\title{
IDENTIFIABILITY OF HOMOGENEOUS POLYNOMIALS AND CREMONA TRANSFORMATIONS
}

\author{
FRANCESCO GALUPPI AND MASSIMILIANO MELLA
}

\begin{abstract}
A homogeneous polynomial of degree $d$ in $n+1$ variables is identifiable if it admits a unique additive decomposition in powers of linear forms. Identifiability is expected to be very rare. In this paper we conclude a work started more than a century ago and we describe all values of $d$ and $n$ for which a general polynomial of degree $d$ in $n+1$ variables is identifiable. This is done by classifying a special class of Cremona transformations of projective spaces.
\end{abstract}

\section{INTRODUCTION}

In this paper we are interested in additive decompositions of homogeneous polynomials. This is usually called Waring problem, after 1770 Waring statement on additive decomposition of integers in additive powers. Let $F \in \mathbb{C}\left[x_{0}, \ldots, x_{n}\right]_{d}$ be a general homogeneous polynomial of degree $d$. The additive decomposition we are looking for is

$$
F=L_{1}^{d}+\ldots+L_{h}^{d}
$$

where $L_{i} \in \mathbb{C}\left[x_{0}, \ldots, x_{n}\right]_{1}$ are linear forms. The problem is classical. The first results are due to Sylvester [Sy, Hilbert [Hi], Richmond [Ri], and Palatini [Pa], among others. The problem addressed was to find special values of $d$ and $n$ for which the decomposition is unique. When this happens the decomposition yields a canonical form of a general polynomial. In modern terminology this is called $h$ identifiability. Applications of identifiability range from Blind Signal Separation to Phylogenetic and Algebraic statistic, see [Lan for an account of applications, and it is studied for both polynomials and tensors, see for instance [COV1] AHJKS. It is expected that canonical forms are a rare phenomenon, see conjectures in [HOOS]. The following is the list of known identifiable cases. A general form $f$ of degree $d$ in $n+1$ variables is $s$-identifiable in the following cases:

- $n=1, d=2 k-1$ and $s=k$, Sy

- $n=3, d=3$ and $s=5$ Sylvester's Pentahedral Theorem, Sy

- $n=2, d=5$ and $s=7[\mathrm{Hi}$, $\mathrm{Ri}$, $\mathrm{Pa}$, see also [MM] for a different approach.

All these cases where known a century ago and were expected to be the only ones, $\mathrm{Br}$. Only quite recently a significant result was obtained in [Me1] where the author proved that these are the only cases if either $d>n$ or $n \leq 3$.

In this paper we complete the study of identifiability proving that these are the only identifiable polynomials.

Date: September 2017.

1991 Mathematics Subject Classification. Primary 14J70 ; Secondary 14N05, 14 E05.

Key words and phrases. Waring, identifiable, linear system, singularities, birational maps.

Partially supported by Progetto MIUR "Geometria sulle varietà algebriche". 
Theorem 1. Let $f$ be a general homogeneous form of degree $d$ in $n+1$ variables. Then $f$ is $s$-identifiable if and only if $(n, d, s)=(1,2 k-1, k),(3,3,5),(2,5,7)$.

Despite its algebraic statement we approach the problem from a birational geometry point of view. The starting point is [Me1, Theorem 2.1] where it is proved that identifiability forces a particular tangential projection of the Veronese variety to be birational. This projection is associated to linear systems with imposed singularities. Then our main result is a consequence of the following statement about Cremona modifications of $\mathbb{P}^{n}$, which is of interest in itself.

Theorem 2. Let $\mathcal{L}_{n, d}\left(2^{h}\right) \subset\left|\mathcal{O}_{\mathbb{P}^{n}}(d)\right|$ be the linear system of forms of degree $d$ with $h$ double points in general position and $\varphi_{n, d, h}$ the rational map associated to it. Then $\varphi_{n, d, h}$ is a Cremona transformation, i.e. $\operatorname{dim} \mathcal{L}_{n, d}\left(2^{h}\right)=n$ and $\varphi_{n, d, h}: \mathbb{P}^{n} \rightarrow \mathbb{P}^{n}$ is birational, if and only if

- $n=1, d=2 k+1$, and $h=k$,

- $n=2, d=5$ and $h=6$,

- $n=3, d=3$ and $h=4$.

The main difficulty in proving Theorem 2 is to control the singularities and the base locus of the linear system $\mathcal{L}_{n, d}\left(2^{h}\right)$. The first task is accomplished in Me1, Corollary 4.5]. It is proved that, for $d \geq 4$, the singularities of $\mathcal{L}_{n, d}\left(2^{h}\right)$ are only the double points imposed, the degree 3 case has been recently completed by COV2]. This allowed to conclude in the mentioned range. Unfortunately if $d \leq n$ it is necessary to control not only the singularities but also the base locus of these linear systems to bound the degree of the map. In Me2 some special cases were proved assuming a divisibility condition on the degree. Here we approach the problem from a different perspective. Instead of trying to bound directly the degree of the map associated to $\mathcal{L}_{n, d}\left(2^{h}\right)$ we produce a degeneration of the imposed singularities in such a way that the limit linear system admits a hyperplane on which the restricted map is still expected to be non birational and then proceed by induction trying to bound the sectional genus of the linear systems we are considering.

This reminds the techniques of interpolation. Indeed the linear systems we are interested in have been studied for the interpolation problem and we profit both of Alexander-Hirschowitz' paper $[\mathrm{AH}]$ and of more recent approaches due to Postinghel Pos2 and Brambilla-Ottaviani [BO]. The proof of Theorem 2 is done by induction on $n$. The induction step is done via a careful choice of numbers. This numerology is the core of the (differentiable) Horace method in $\mathrm{AH}$, where it is played a double induction on both degree and dimension. We were not able to control the sectional genus along these specializations. For this reason we have to develop a different approach based only on dimension induction. We let some double points collapse into a 3 -ple point with tangent directions. The latter allows us to make induction work and to study the restriction of the linear system to a hyperplane.

This leads us to study the standard interpolation problems for linear systems with one triple point, with tangent directions in general position, and a bunch of double points. The first step of induction is the study of planar linear systems. Here we benefit from the theory developed around Harbourne-Hirschowitz conjecture, see Cil, in particular we use the results in [CM] about quasi-homogeneous multiplicity. As usual in interpolation problems, for low degrees $d \leq 5$ and in particular for cubics, we need special arguments. Once we worked out the case with tangent direction in general position we extend it to the set of tangent direction arising in the flat limit of $(n+1)$ double points that collapse to a point of multiplicity 3 concluding the proof. 
Here is an outline of the paper. In section 1 we introduce the notation we use and describe the reduction from Theorem 11 to Theorem 2 , In section 2 we prove the result we need about degeneration with and without collapsing points. In particular we compute the limits of some special configurations of double points. We were not able to find the theorems we needed stated explicitly, therefore we proved everything but this section is inspired by Nesci's and Postinghel's theses, Ne Pos1. In section 3 we prove the main induction argument. This is done in several steps to help, at least this is our hope, the reader to digest all the numerology needed. In section 4 we bound the sectional genus of the linear system we are interested in. In section 5 we apply the result obtained to the flat limit of $(n+1)$ collapsing double points, settle the special case of cubics and prove Theorem 2 .

Acknowledgments We are grateful to the referees for a careful reading, for suggestions that improves the expositions, and for pointing out wrong computations in the first version of the paper.

\section{Notations AND PRELIMINARIES}

We work over the complex field.

Definition 3. Let $Z$ be a 0 -dimensional scheme. The degree, or length, of $Z$, denoted by $\operatorname{deg} Z$, is the dimension of its ring of regular functions as a complex vector space.

We start recalling some useful facts about 0-dimensional schemes.

Proposition 4. Let $Z$ be a 0 -dimensional subscheme.

i) The degree of $Z$ is the value of the Hilbert polynomial of $Z$.

ii) Let $X, Y$ be 0 -dimensional schemes such that $X \subseteq Y$ and $\operatorname{deg} X=\operatorname{deg} Y$. Then $X=Y$.

We deal with linear systems on $\mathbb{P}^{n}$ with assigned singularities and tangent directions.

Notation 5. Let $\left\{p_{1}, \ldots, p_{r}\right\} \subset \mathbb{P}^{n}$ be a set of points and $\left\{q_{1}, \ldots, q_{j}\right\} \in \mathbb{P}\left(\mathbb{T}_{p_{1}} \mathbb{P}^{n}\right)$ a set of tangent directions (infinitely near points) in $p_{1}$. The linear system

$$
\mathcal{L}_{n, d}\left(m_{1}, \ldots, m_{r}\right)\left(p_{1}\left[\left\{q_{1}, \ldots, q_{j}\right\}\right], p_{2}, \ldots, p_{r}\right) \subset\left|\mathcal{O}_{\mathbb{P}^{n}}(d)\right|
$$

is the sublinear system of hypersurfaces having multiplicities $m_{i}$ at the point $p_{i}$ and whose tangent cone at $p_{1}$ contains $\left\{q_{1}, \ldots, q_{j}\right\}$. If either the points $\left\{p_{1}, \ldots, p_{r}\right\}$ and $\left\{q_{1}, \ldots, q_{j}\right\}$ are in general position or no confusion is likely to arise, we indicate

$$
\mathcal{L}_{n, d}\left(m_{1}[j], m_{2} \ldots, m_{r}\right):=\mathcal{L}_{n, d}\left(m_{1}, \ldots, m_{r}\right)\left(p_{1}\left[\left\{q_{1}, \ldots, q_{j}\right\}\right], p_{2}, \ldots, p_{r}\right)
$$

and

$$
\mathcal{L}_{n, d}\left(m_{1}, \ldots, m_{r}\right):=\mathcal{L}_{n, d}\left(m_{1}[0], m_{2} \ldots, m_{r}\right) .
$$

Moreover if $m_{1}=\ldots=m_{g}=m$ we indicate

$$
\mathcal{L}_{n, d}\left(m^{g}, m_{g+1}, \ldots, m_{r}\right):=\mathcal{L}_{n, d}\left(m_{1}, \ldots, m_{r}\right) .
$$

Definition 6. The virtual dimension of such a linear system is

$$
\operatorname{virtdim} \mathcal{L}_{n, d}\left(m_{1}[j], m_{2} \ldots, m_{r}\right)=\left(\begin{array}{c}
d+n \\
n
\end{array}\right)-1-\sum_{i=1}^{r}\left(\begin{array}{c}
m_{i}-1+n \\
n
\end{array}\right)-j,
$$

the expected dimension is defined as

$$
\operatorname{expdim} \mathcal{L}_{n, d}\left(m_{1}[j], \ldots, m_{r}\right)=\max \left\{\operatorname{virtdim} \mathcal{L}_{n, d}\left(m_{1}[j], m_{2} \ldots, m_{r}\right),-1\right\},
$$


where dimension -1 indicates that the linear system is expected to be empty. Note that

$$
\operatorname{dim} \mathcal{L}_{n, d}\left(m_{1}[j], m_{2} \ldots, m_{r}\right) \geq \operatorname{expdim} \mathcal{L}_{n, d}\left(m_{1}[j], m_{2} \ldots, m_{r}\right) .
$$

If $\operatorname{dim} \mathcal{L}_{n, d}\left(m_{1}[j], m_{2} \ldots, m_{r}\right)>\operatorname{dim} \mathcal{L}_{n, d}\left(m_{1}[j], m_{2} \ldots, m_{r}\right)$, then the linear system is said to be special. Otherwise it is called nonspecial.

The speciality of linear systems has been extensively studied, see Cil for an account, but very little is known in general. The better understood cases are linear systems with only double points and linear systems of plane curves.

If all $m_{i}=2$ there is the famous Alexander-Hirschowitz theorem.

Theorem 7. $\mathrm{AH} \mathcal{L}_{n, d}\left(2^{h}\right)$ is special if and only if $(n, d, h)$ is one of the following:

i) $(n, 2, h)$ with $2 \leq h \leq n$,

ii) $(2,4,5),(3,4,9),(4,3,7),(4,4,14)$.

Remark 8. Further note that for all special linear systems in Theorem 7 ii) the virtual dimension is negative while the dimension is 0 .

For linear systems of plane curves there is a very precise conjecture about speciality. Since we are going to use some of its known cases in section 4 we collect here the necessary notation and statement.

Let $\left\{p_{1}, \ldots, p_{r}\right\} \subset \mathbb{P}^{2}$ be general points, $\nu: \tilde{\mathbb{P}}^{2} \rightarrow \mathbb{P}^{2}$ their blow up, and $\mathcal{L}:=$ $\mathcal{L}_{2, d}\left(m_{1}, \ldots, m_{r}\right)\left(p_{1}, \ldots, p_{r}\right)$. We put a tilde to indicate the strict transform of curves on $\tilde{\mathbb{P}}^{2}$.

Definition 9. A $(-1)$-curve $C \subset \mathbb{P}^{2}$ is a curve such that $\tilde{C}$ is a smooth rational curve with self intersection -1 .

The linear system $\mathcal{L}$ on $\mathbb{P}^{2}$ is $(-1)$-reducible if

$$
\mathcal{L}=\sum_{i=1}^{k} N_{i} C_{i}+\mathcal{M},
$$

where $C=\sum_{i=1}^{k} C_{i}$ is a configuration of $(-1)$ curves, $\tilde{\mathcal{M}} \cdot \tilde{C}_{i}=0$, for all $i=1, \ldots, k$, and $\operatorname{virtdim}(\mathcal{M}) \geq 0$.

The system $\mathcal{L}$ is called $(-1)$-special if, in addition, there is an $i \in\{1, \ldots, k\}$ such that $N_{i}>1$.

The leading conjecture for linear systems of plane curves has been formulated by Harbourne and Hirschowitz in 1989.

Conjecture 10 (Harbourne-Hirschowitz). A linear system of plane curves is special if and only if it is $(-1)$-special.

The conjecture is known to be true in some cases and in particular CilibertoMiranda, [CM], proved it for $\mathcal{L}_{2, d}\left(n, m^{h}\right)$ for $m \leq 3$.

The sectional genus of a linear system is the geometric genus of a general curve section. We will prove that the linear systems we are interested in have positive sectional genus. For this purpose the following remark is extremely useful.

Remark 11. The genus of any curve of an algebraic system of algebraic curves is not greater than the genus of the generic curve of the system, $\mathrm{Ch}$. Therefore to prove that a linear system $\mathcal{L}$ has positive sectional genus it is enough to show a curve of positive genus in some algebraic family of curves whose general member is a curve section of $\mathcal{L}$.

We now reduce Theorem 1 to Theorem 2 following [Me1]. Let $n, d$ be integers. Then a general polynomial $F \in \mathbb{C}\left[x_{0}, \ldots, x_{n}\right]_{d}$ admits a unique decomposition

$$
F=L_{1}^{d}+\ldots+L_{s}^{d}
$$


with $L_{i} \in \mathbb{C}\left[x_{0}, \ldots, x_{n}\right]_{1}$, if and only if the $s$-secant map $\pi_{s}: \sec _{s}\left(V_{d, n}\right) \rightarrow \mathbb{P}^{N}$ of the Veronese variety is dominant and birational, where $N=\left(\begin{array}{c}n+d \\ n\end{array}\right)-1$ and $\sec _{s}\left(V_{d, n}\right)$ is the abstract $s$-secant variety. Since $\operatorname{dim}_{\sec }\left(V_{d, n}\right)=s(n+1)-1$, for $\pi_{s}$ to be birational there is a numerical constrain. That is

$$
k(n, d):=\frac{\left(\begin{array}{c}
d+n \\
n
\end{array}\right)}{n+1}
$$

has to be an integer. Now let $\operatorname{Sec}_{s}\left(V_{n, d}\right)$ be the embedded $s$-secant variety. For a general point $z \in \operatorname{Sec}_{k(n, d)-1}\left(V_{n, d}\right)$, let $\varphi: \mathbb{P}^{N} \rightarrow \mathbb{P}^{n}$ be the projection from the embedded tangent space $\mathbb{T}_{z} \operatorname{Sec}_{k(n, d)-1}\left(V_{n, d}\right)$. In [Me1, Theorem 2.1] it is proved that if $\pi_{k(n, d)}$ is birational then $\varphi_{\mid V_{n, d}}$ is birational. It is well known, see for instance Me1, that, by Terracini Lemma, this map is associated to the linear system $\mathcal{L}_{n, d}\left(2^{k(n, d)-1}\right)$. Therefore the morphism $\pi_{k(n, d)}$ is birational only if the map associated to $\mathcal{L}_{n, d}\left(2^{k(n, d)-1}\right)$ is birational. Using this, Theorem 1 is a consequence of Theorem 2 .

\section{Limit OF DOUBLE POINTS}

A standard approach to study the speciality of linear systems $\mathcal{L}_{n, d}\left(m_{1}, \ldots, m_{r}\right)$ is via degeneration. This is accomplished by using a flat family in which the involved points specialize in some special configuration. Often some of the points are sent on a hyperplane to apply induction arguments. In our construction we need several degenerations and, unlike the usual set up that concerns degeneration of points in general positions to special position, we need to further degenerate also special positions. Thanks to ii) in Proposition 4 we may study specializations in a local setup. We set some notation that will be used throughout the paper.

Definition 12. A degeneration is a morphism $\pi: V \rightarrow \Delta$, where $\Delta \ni 0,1$ is a complex disk, $V$ is a smooth variety and $\pi$ is proper and flat. For any $t \in \Delta$ we denote the fiber of $\pi$ over $t$ by $V_{t}$. Let $\sigma_{i}: \Delta \rightarrow V$ be sections of $\pi$ and $Z$ a scheme with $Z_{\text {red }}=\cup_{i} \sigma_{i}(\Delta)$. We let $Z_{t}:=Z \cap V_{t}$, for $t \neq 0$, and $Z_{0}$ their flat limit. We say that $Z_{0}$ is a specialization of $Z_{t}$.

In this paper we are mainly interested in the following case of specialization.

Construction 13 (Specialization without collisions). Let $X$ be the blow up of $\mathbb{P}^{n}$ in the point $p_{1}$, with exceptional divisor $E, V:=X \times \Delta$, and $\pi: V \rightarrow \Delta$ the canonical projection. Fix $j$ disjoint sections $\left\{\tau_{1}, \ldots \tau_{j}\right\}$ such that $\tau_{i}(\Delta) \subset E \times \Delta$ and $r-1$ disjoint sections $\left\{\sigma_{2}, \ldots, \sigma_{r}\right\}$ such that $\sigma_{i}(\Delta) \cap(E \times \Delta)=\emptyset$. Let

$$
Z:=\bigcup_{i=2}^{r} \sigma_{i}(\Delta)^{m_{i}} \cup \bigcup_{h=1}^{j} \tau_{h}(\Delta)
$$

be the scheme supported on the sections with multiplicity $m_{i}$ along $\sigma_{i}(\Delta)$. Let

$$
\mathbb{L}_{n, d}\left(m_{1}[j], m_{2} \ldots, m_{r}\right)\left(p_{1}\left[\left\{\tau_{1}, \ldots, \tau_{j}\right\}\right], \sigma_{2}, \ldots, \sigma_{r}\right)
$$

be the linear subsystem on $V$ associated to divisors of degree $d$ having multiplicities $m_{i}$ along $\sigma_{i}(\Delta), m_{1}$ in $p_{1}$ and whose tangent cone contains $\tau_{j}(\Delta)$. Then for any $t \in \Delta$ the linear system

$$
\mathbb{L}_{n, d}\left(m_{1}[j], m_{2}, \ldots, m_{r}\right)\left(p_{1}\left[\left\{\tau_{1}, \ldots, \tau_{j}\right\}\right], \sigma_{2}, \ldots, \sigma_{r}\right)_{\mid V_{t}}
$$

is

$$
\mathcal{L}_{t}:=\mathcal{L}_{n, d}\left(m_{1}[j], m_{2}, \ldots, m_{r}\right)\left(p_{1}\left[\left\{\tau_{1}(t), \ldots, \tau_{j}(t)\right\}\right], \sigma_{2}(t), \ldots, \sigma_{r}(t)\right) .
$$

By semicontinuity we have

$$
\mathrm{h}^{0}\left(V_{0}, \mathcal{L}_{0}\right) \geq \mathrm{h}^{0}\left(V_{t}, \mathcal{L}_{t}\right) .
$$


Therefore to prove the nonspeciality of $\mathcal{L}_{t}$ it is enough to produce a specialization having $\mathcal{L}_{0}$ nonspecial.

Definition 14. Let $Z_{0}$ be a specialization of $Z_{1}$ as in Construction 13. Then we say that the linear system $\mathcal{L}_{0}$ is a specialization of $\mathcal{L}_{1}$.

Remark 15. Let $H \subset \mathbb{P}^{n}$ be a hyperplane through the point $p_{1}$ and $Z_{1}:=$ $\left\{p_{2}^{m_{2}}, \ldots, p_{s}^{m_{s}}\right\} \cup\left\{t_{1}, \ldots, t_{j}\right\}$ a 0 -dimensional scheme as in Construction 13. Assume that $\left\{p_{2}, \ldots, p_{h}\right\} \subset H$ and $\left\{t_{1}, \ldots, t_{l}\right\} \subset \mathbb{T}_{p_{1}} H$, then a classical way to study special linear system is via the Castelnuovo exact sequence that in this case reads

$$
\begin{array}{r}
0 \rightarrow \mathcal{L}_{n, d-1}\left(\left(m_{1}-1\right)[j-l], m_{2}-1, \ldots, m_{h}-1, m_{h+1}, \ldots, m_{s}\right) \rightarrow \\
\rightarrow \mathcal{L}_{n, d}\left(m_{1}[j], m_{2}, \ldots, m_{s}\right) \rightarrow \mathcal{L}_{n-1, d}\left(m_{1}[l], m_{2}, \ldots, m_{h}\right) .
\end{array}
$$

Therefore the nonspeciality of the linear systems

$\mathcal{L}_{n, d-1}\left(\left(m_{1}-1\right)[j-l], m_{2}-1, \ldots, m_{h}-1, m_{h+1}, \ldots, m_{s}\right)$ and $\mathcal{L}_{n-1, d}\left(m_{1}[l], m_{2}, \ldots, m_{h}\right)$ implies the nonspeciality of $\mathcal{L}_{n, d}\left(m_{1}[j], m_{2}, \ldots, m_{s}\right)$.

We state for future reference the following well known fact.

Lemma 16. Let $\mathcal{L}$ be a linear system on a smooth projective variety $X$ and $C \subset X$ a positive dimensional subvariety. Assume $\operatorname{cod}_{\mathcal{L}}\left|\mathcal{L} \otimes \mathcal{I}_{C}\right|=$ s. Set $x_{1}, \ldots, x_{s} \in C$ general points, then $x_{1}, \ldots, x_{s}$ impose independent conditions to $\mathcal{L}$.

We apply the above remarks to prove the nonspeciality of some linear systems we will use along the proof of Theorem 1.

Proposition 17. Let $n \geq 3$ and $d \geq 4$, define

$$
r(n, d):= \begin{cases}\left\lceil\frac{\left(\begin{array}{c}
n+d \\
n
\end{array}\right)}{n+1}\right\rceil-n-1 & \text { if either } n \neq 3 \text { or }(n, d)=(3,4) ; \\
\left\lceil\frac{\left(\begin{array}{c}
+3 \\
3
\end{array}\right)}{4}\right\rceil-5 & \text { if } n=3 .\end{cases}
$$

The linear system $\mathcal{L}_{n, d}\left(3,2^{a}\right)$ is nonspecial if $a \leq r(n, d)$.

Proof. We prove the statement by induction on $d$. It is clear that it is enough to prove it for $a=r(n, d)$. The first step of induction is $d=4$ and it is the content of Pos2, Lemma 2.4].

Assume $d \geq 5$. Let $Z_{1}:=\left\{q^{3}, p_{1}^{2}, \ldots, p_{a}^{2}\right\}$ be a 0 -dimensional scheme. Fix a hyperplane $H$ not containing $q$. Let $Z_{0}$ be a specialization without collisions of $Z_{1}$ with

$$
h:=\left\lceil\frac{\left(\begin{array}{c}
d+n-1 \\
n-1
\end{array}\right)}{n}\right\rceil-1
$$

points on the hyperplane $H$. Since $q \notin H$ the Castelnuovo exact sequence reads

$$
0 \rightarrow \mathcal{L}_{n, d-1}\left(3,2^{a-h}, 1^{h}\right) \rightarrow \mathcal{L}_{n, d}\left(3,2^{a}\right) \rightarrow \mathcal{L}_{n-1, d}\left(2^{h}\right),
$$

where the simple base points are all on the hyperplane $H$. Since $d \geq 5$ the linear system on the right is nonspecial by Theorem 7 . Therefore to conclude it is enough to prove that $\mathcal{L}_{n, d-1}\left(3,2^{a-h}, 1^{h}\right)$ is nonspecial.

Claim 1. expdim $\mathcal{L}_{n, d-1}\left(3,2^{a-h}, 1^{h}\right)$ is non negative.

Proof. Assume first that $n>3$. Then

$\operatorname{expdim} \mathcal{L}_{n, d-1}\left(3,2^{a-h}, 1^{h}\right)=\left(\begin{array}{c}n+d-1 \\ n\end{array}\right)-\left(\begin{array}{c}n+2 \\ 2\end{array}\right)-(n+1) a+n h-1 \geq n^{2}-\left(\begin{array}{c}n+2 \\ 2\end{array}\right)-1 \geq 0$ 
Assume that $n=3$. Then

$$
\operatorname{expdim} \mathcal{L}_{3, d-1}\left(3,2^{a-h}, 1^{h}\right)=\left(\begin{array}{c}
d+2 \\
3
\end{array}\right)-10-4 a+3 h-1 \geq 16-14>0
$$

We start proving that the simple base points impose independent conditions. The points are general in $H$, therefore, by Lemma 16, we have to check that

$$
\operatorname{dim}\left|\mathcal{L}_{n, d-2}\left(3,2^{a-h}\right)\right| \leq 0
$$

This is clear for $d=4$. For $d \geq 5$ by Theorem 7 , checking also the special cases, we have

$\operatorname{dim} \mathcal{L}_{n, d-2}\left(3,2^{a-h}\right) \leq \operatorname{dim} \mathcal{L}_{n, d-2}\left(2^{a-h+1}\right)=\left(\begin{array}{c}n+d-2 \\ n\end{array}\right)-1-(n+1)(a-h+1)$.

For $n=3$ this reads

$$
\begin{aligned}
\operatorname{dim} \mathcal{L}_{n, d-2}\left(3,2^{a-h}\right) & \leq\left(\begin{array}{c}
d+1 \\
3
\end{array}\right)-4\left(\left\lceil\frac{\left(\begin{array}{c}
d+3 \\
3
\end{array}\right)}{4}\right\rceil-5-\left\lceil\frac{\left(\begin{array}{c}
d+2 \\
2
\end{array}\right)}{3}\right\rceil+2\right)-1 \\
& \leq\left(\begin{array}{c}
d+1 \\
3
\end{array}\right)-\left(\begin{array}{c}
d+3 \\
3
\end{array}\right)+\frac{4}{3}\left(\begin{array}{c}
d+2 \\
2
\end{array}\right)+15 \\
& =-\left(\begin{array}{c}
d+1 \\
2
\end{array}\right)+\frac{1}{3}\left(\begin{array}{c}
d+2 \\
2
\end{array}\right)+15=\frac{1-d^{2}}{3}+15<0
\end{aligned}
$$

for every $d \geq 7$. We check the rest of the cases by a direct computation.

$(n, d)=(3,5)$ In this case $a=9$ and $h=6$ and the linear system $\mathcal{L}_{3,3}\left(3,2^{3}\right)$ has a unique element.

$(n, d)=(3,6)$ In this case $a=16$ and $h=9$. An element of the linear system $\mathcal{L}_{3,4}\left(3,2^{7}\right)$ has to contain all quadrics passing through the 8 singular points. This shows that $\mathcal{L}_{3,4}\left(3,2^{7}\right)$ is empty.

Inequality (2) for $n>3$ yields

$$
\begin{aligned}
\operatorname{dim} \mathcal{L}_{n, d-2}\left(3,2^{a-h}\right) & \leq\left(\begin{array}{c}
n+d-2 \\
n
\end{array}\right)-(n+1)\left(\left\lceil\frac{\left(\begin{array}{c}
n+d \\
n
\end{array}\right)}{n+1}\right\rceil-\left\lceil\frac{\left(\begin{array}{c}
n+d-1 \\
n-1
\end{array}\right)}{n}\right\rceil-n+1\right)-1 \\
& \leq\left(\begin{array}{c}
n+d-2 \\
n
\end{array}\right)-\left(\begin{array}{c}
n+d \\
n
\end{array}\right)+\left(\begin{array}{c}
n+d-1 \\
n-1
\end{array}\right)+\frac{\left(\begin{array}{c}
n+d-1 \\
n-1
\end{array}\right)}{n}+n^{2}+n-1 \\
& =-\left(\begin{array}{c}
n+d-2 \\
n-1
\end{array}\right)+\frac{\left(\begin{array}{c}
n+d-1 \\
n-1
\end{array}\right)}{n}+n^{2}+n-1 \\
& =-\frac{(n+d-2) !}{(n-1) !(d-1) !}+\frac{(n+d-1) !}{n ! d !}+n^{2}+n-1 \\
& =\frac{(n+d-2) !}{(n-1) !(d-1) !}\left[\begin{array}{c}
n+\frac{n+d-1}{n d} \\
\left(n-1+n^{2}+n-1\right.
\end{array}\right. \\
& =\left(\begin{array}{c}
n+d-2 \\
n-1
\end{array}\right) \cdot \frac{(1-n)(d-1)}{n d}+n^{2}+n-1 .
\end{aligned}
$$


The latter decreases as $d$ increases, so

for every $n>3$.

$$
\begin{aligned}
\operatorname{dim} \mathcal{L}_{n, d-2}\left(3,2^{a-h}\right) & \leq\left(\begin{array}{c}
n+d-2 \\
n-1
\end{array}\right) \cdot \frac{(1-n)(d-1)}{n d}+n^{2}+n-1 \\
& \leq \frac{4}{5}\left(\begin{array}{c}
n+3 \\
4
\end{array}\right) \cdot \frac{1-n}{n}+n^{2}+n-1 \\
& =\frac{-n^{4}-5 n^{3}+25 n^{2}+35 n-24}{30}<0
\end{aligned}
$$

To conclude we prove the nonspeciality of $\mathcal{L}_{n, d-1}\left(3,2^{a-h}\right)$. For this observe that

$$
\begin{aligned}
a-h-r & (n, d-1) \leq \frac{\left(\begin{array}{c}
n+d \\
n
\end{array}\right)}{n+1}-\frac{\left(\begin{array}{c}
d+n-1 \\
n-1
\end{array}\right)}{n}-\frac{\left(\begin{array}{c}
n+d-1 \\
n
\end{array}\right)}{n+1}+2 \\
& =\frac{1}{n(n+1)}\left[n\left(\begin{array}{c}
n+d \\
n
\end{array}\right)-(n+1)\left(\begin{array}{c}
d+n-1 \\
n-1
\end{array}\right)-n\left(\begin{array}{c}
n+d-1 \\
n
\end{array}\right)\right]+2 \\
& =-\frac{\left(\begin{array}{c}
n+d-1 \\
n-1
\end{array}\right)}{n(n+1)}+2<1
\end{aligned}
$$

Therefore we conclude by induction hypothesis.

Lemma 18. Let $\frac{\left(\begin{array}{c}d+3 \\ 3\end{array}\right)}{4}-1>b \geq 1$ and $d \geq 4$ be integers. Fix $q \in \Pi \subset \mathbb{P}^{n}$ a linear space of dimension 3 and $b$ general points on it, say $x_{1}, \ldots, x_{b}$. Then the linear system

is nonspecial if

$$
\mathcal{L}_{n, d}\left(2^{a}, 1^{b}\right)\left(q, p_{1}, \ldots, p_{a-1}, x_{1}, \ldots, x_{b}\right)
$$

$$
a \leq a(n, d):=\left\lfloor\frac{\left(\begin{array}{c}
n+d \\
n
\end{array}\right)-b-1}{n+1}\right\rfloor-\max \{0, n-4\},
$$

where the $p_{i}$ are general points. For the special case $(d, b)=(4,5)$ we prove a better estimate

$$
a(n, 4)=\left\lfloor\frac{\left(\begin{array}{c}
n+4 \\
n
\end{array}\right)-5-1}{n+1}\right\rfloor-\max \{(n-7), 1\}
$$

Proof. Note that since $b \geq 1, d \geq 4$ and the virtual dimension is non negative then $\mathcal{L}_{n, d}\left(2^{a}\right)$ is nonspecial by Theorem 7 . Therefore we have only to care about the simple points.

For $n=3$ the statement is immediate. For $n=4$, by Lemma 16, it is enough to check that $\operatorname{dim} \mathcal{L}_{4, d-1}\left(2^{a(4, d)-1}, 1\right) \leq 0$. By Theorem 7 it is enough to check that $\operatorname{virtdim} \mathcal{L}_{4, d-1}\left(2^{a(4, d)-1}, 1\right)<0$. The latter is a simple computation.

Next we prove the statement by induction on $n$. For $n=i+1 \geq 5$ fix a general hyperplane $H \supset \Pi$ and consider a degeneration with $a(i, d)$ points on $H$. Then, by Castelnuovo exact sequence, we have to prove the nonspeciality of

$$
\mathcal{L}_{i, d}\left(2^{a(i, d)}, 1^{b}\right), \text { and } \mathcal{L}_{i+1, d-1}\left(2^{a(i+1, d)-a(i, d)}, 1^{a(i, d)}\right)
$$

the former is nonspecial by the induction step. For the latter note that

$$
\begin{aligned}
a(i+1, d)-a(i, d) & \geq \frac{\left(\begin{array}{c}
i+1+d \\
i+1
\end{array}\right)-b-1}{i+2}-\frac{\left(\begin{array}{c}
i+d \\
i
\end{array}\right)-b-1}{i+1}-2 \\
& >\frac{\left(\begin{array}{c}
i+1+d \\
i+1
\end{array}\right)-b-1}{i+2}-\frac{\left(\begin{array}{c}
i+d \\
i
\end{array}\right)-b-1}{i+2}-2 \\
& >\frac{\left(\begin{array}{c}
i+d \\
i+1
\end{array}\right)}{i+2}-2 \geq \frac{\left(\begin{array}{c}
i+d-1 \\
i+1
\end{array}\right)}{i+2} .
\end{aligned}
$$


For $d \geq 5$ by Theorem 7 the linear system $\mathcal{L}_{i+1, d-2}\left(2^{a(i+1, d)-a(i, d)}\right)$ is empty. For $d=4$ it is easy to see that

$$
a(i+1,4)-a(i, 4)>i
$$

and again $\mathcal{L}_{i, 2}\left(2^{a(i+1,4)-a(i, 4)}\right)$ is empty. Let $a(i, d)=\left\lfloor\frac{\left(\begin{array}{c}i+d \\ i\end{array}\right)-b-1}{i+1}\right\rfloor-\alpha(i)$, then $\alpha(i+1)=\alpha(i)+1$. Then we have

$\left.\operatorname{virtdim} \mathcal{L}_{i+1, d-1}\left(2^{a(i+1, d)-a(i, d)}, 1^{a(i, d)}\right) \geq(i+2) \alpha(i+1)-(i+1)(\alpha(i)+1)\right)-1>0$

and Lemma 16 proves that the linear system $\mathcal{L}_{i+1, d-1}\left(2^{a(i+1, d)-a(i, d)}, 1^{a(i, d)}\right)$ is non special to conclude this case.

Assume that $d=4$ and $b=5$. We first prove that $\mathcal{L}_{5,4}\left(2^{19}, 1^{5}\right)$ is non special. Observe that degenerating 12 double points on a hyperplane by Castelnuovo exact sequence we have that $\mathcal{L}_{5,4}\left(2^{19}\right)$ decomposes in $\mathcal{L}_{4,4}\left(2^{12}\right)$ and $\mathcal{L}_{5,3}\left(2^{7}, 1^{12}\right)$. It is easy to check that these two linear systems are non special and $\operatorname{dim} \mathcal{L}_{5,3}\left(2^{7}, 1^{12}\right)=$ 1. The linear system $\mathcal{L}_{4,3}\left(2^{11}\right)$ is empty therefore there is at most a pencil of divisors in $\mathcal{L}_{5,4}\left(2^{19}\right)$ that contains a given $\mathbb{P}^{3}$ through a double point. By hypothesis $\operatorname{virtdim} \mathcal{L}_{5,4}\left(2^{19}, 1^{5}\right)>1$ hence this linear system is not special. To conclude the statement for $d=4$ we then argue exactly as in the first part of the proof, checking Equation (3) case by case for $n \leq 8$ and then conclude as in the general case.

At the first stage of our proof we need to allow some points to collapse.

Construction 19 (Specialization with $h$ collapsing points). Let $V=\mathbb{A}^{n} \times \Delta$ and $\pi: V \rightarrow \Delta$ be the canonical projection. Fix $h$ general sections $\left\{\sigma_{1}, \ldots, \sigma_{h}\right\}$ such that $\sigma_{i}(0)=q$. Let $Z:=\cup_{i} \sigma_{i}(\Delta)^{m_{i}}$ and $\nu: V \rightarrow \mathbb{A}^{n}$ be the projection.

Let $X \rightarrow V$ be the blow up of $V$ at the point $q$, with exceptional divisor $W$. Then we have natural morphisms $\nu_{X}: X \rightarrow \mathbb{A}^{n}$, a degeneration $\pi_{X}: X \rightarrow \Delta$, and sections $\sigma_{X, i}: \Delta \rightarrow X$. The fiber $X_{0}$ is given by $W \cup V_{0}$, where $V_{0}$ is $\mathbb{A}^{n}$ blown up in one point and $W \cong \mathbb{P}^{n}$. Let $R=W \cap V_{0} \cong \mathbb{P}^{n-1}$ be the exceptional divisor of this blow up. We want to stress that since the sections $\sigma_{i}$ 's are general $\left\{\sigma_{X, i}(0)\right\}$ are general points of $W$.

Our main concern is to determine $Z_{0}$ the flat limit of $Z_{t}:=Z_{\mid V_{t}}$ in Construction 19. This is in general quite hard and we only have partial answers to this question. Nonetheless once we understand the limit, we may study the speciality of a linear system via its specializations with collapsing points, using the same technique described in Construction 13 and Remark [15.

Lemma 20. The multiplicity in $q$ of $Z_{0}$ is at least the minimum integer $j$ such that the linear system $\mathcal{L}_{n, j}\left(m_{1}, \ldots, m_{h}\right)$ is not empty.

Proof. By definition we have

$$
\operatorname{mult}_{q} Z_{0} \geq \operatorname{mult}_{q} Z=: \mu \text {. }
$$

Let $\mathcal{I}_{X, Z}$ be the ideal associated to $Z$ on $X$, then $\mathcal{I}_{X, Z \mid W} \sim \mathcal{L}_{n, \mu}\left(m_{1}, \ldots, m_{h}\right)$. The ideal $\mathcal{I}_{Z}$ is globally generated therefore the linear system $\mathcal{I}_{X, Z \mid W}$ has to be non empty.

Due to the lack of a classification of special linear systems, it is in general quite hard to determine the exact value predicted by Lemma 20. In this paper we are only interested in collapsing of double points, the more general case of arbitrary multiplicities will be treated elsewhere. Theorem 7 describes completely the special linear systems of type $\mathcal{L}_{n, d}\left(2^{h}\right)$. 
The next result proves that for double points, outside the small list of special linear systems in Theorem 7 the multiplicity of the limit is the one predicted by Lemma 20.

Corollary 21. Let $\pi$ be a specialization with $h$ collapsing points. Assume that $m_{1}=\cdots=m_{h}=2, h \geq n+1$ and $(n, h) \neq(2,5),(3,9),(4,7),(4,14)$. Then the multiplicity of $Z_{0}$ in $q$ is the minimum integer $j$ such that

$$
\left(\begin{array}{c}
n+j \\
n
\end{array}\right)-h(n+1)>0 .
$$

Proof. As before let $V_{0}$ be the central fiber and $q \in V_{0}$ the collapsing point. Let $Z$ be the scheme supported on the union of $\sigma_{i}(\Delta)$ 's with multiplicity 2 . Let $Z_{0}$ be the flat limit of $Z_{t}$, and $\mu$ the minimum integer such that $\left(\begin{array}{c}n+\mu \\ n\end{array}\right)-h(n+1)>0$. The numerical assumption we made, together with Theorem [7] ensure that $\mu$ is the minimum integer such that $\mathcal{L}_{n, \mu}\left(2^{h}\right)$ is not empty and

$$
\operatorname{dim} \mathcal{L}_{n, \mu}\left(2^{h}\right)=\left(\begin{array}{c}
n+\mu \\
n
\end{array}\right)-h(n+1)>0 .
$$

Lemma 20 tells us mult $Z_{0} \geq \mu$. Further note that $\left(\begin{array}{c}n+\mu \\ n\end{array}\right)$ is also the degree of the $(\mu+1)$-tuple point of $\mathbb{P}^{n}$, and $h(n+1)$ is the degree of the 0-dimensional scheme $Z_{1}$. By flatness the latter is preserved therefore equation (4) shows that $\operatorname{mult}_{q} Z_{0} \leq \mu$.

In some special cases the multiplicity is enough to compute the limit scheme.

Example 22. Let us collapse 7 double points in $\mathbb{P}^{2}$, then $(n, h)=(2,7)$. The scheme consisting of those points has length 21 . Since $h^{0}\left(\mathcal{O}_{\mathbb{P}^{2}}(5)\right)=15$, they cannot lie on a quintic curve. On the other hand, they lie on a sextic, so the minimum degree we are looking for is $\mu=6$. By Corollary 21 the limit scheme contains a 6 -ple point. A 6 -ple point in $\mathbb{P}^{2}$ has length 21 . Then by ii) in Proposition 4 the limit scheme is a 6 -ple point.

Let $(n, h)=(3,5)$. The scheme consisting of those points has length 20 . Since $\mathrm{h}^{0}\left(\mathcal{O}_{\mathbb{P}^{3}}(3)\right)=20$, they cannot lie on a cubic surface. By Corollary 21 the multiplicity we are looking for is 4 . As before observe that a 4-ple point in $\mathbb{P}^{3}$ has length 20 . Then by ii) in Proposition 4 the limit scheme is a 4-ple point.

When the scheme we are specializing does not have the degree of a multiple point this analysis is not enough to determine the limit scheme.

Let us start with a limit scheme $Z_{0} \subset \mathbb{A}^{n}$ and assume we are not in the exceptions of Corollary 21. Therefore the multiplicity mult ${ }_{q} Z_{0}=\mu$ is the minimum number such that $\mathcal{L}_{n, \mu}\left(2^{h}\right)$ is not empty. In the setup of Construction 19 we have a degeneration $\pi_{X}: X \rightarrow \Delta$, with central fiber $X_{0}=W \cup V_{0}, W \cong \mathbb{P}^{n}$ is the exceptional divisor of the blow up of $q$, and $R=W \cap V_{0}$.

Let $\Sigma_{X}$ be the smooth scheme associated to the sections $\sigma_{X, i}(\Delta)$. Let $\mathcal{X} \rightarrow X$ be the blow up of the ideal sheaf $\mathcal{I}_{\Sigma_{X}}$, with exceptional divisors $\mathcal{E}_{i}$, and $\varphi: \mathcal{X} \rightarrow \Delta$ the degeneration onto $\Delta$. Note that this blow up is an isomorphism in a neighborhood of $V_{0}$, then the central fiber is

$$
\mathcal{X}_{0}:=\varphi^{-1}(0)=P \cup V_{0},
$$

where $P$ is the blow up of $W$ in $h$ general points. As before let $R=P \cap V_{0}$.

Then the linear systems we are interested in are $\mathcal{L}:=\mathcal{O}_{\mathcal{X}}\left(-\sum_{i} 2 \mathcal{E}_{i}-\mu P\right)$ and its restrictions $\mathcal{L}_{P}, \mathcal{L}_{R}$, to $P$ and $R$. The linear system $\mathcal{L}$ is complete and we aim to understand when its restrictions stay complete.

Let us start with an instructive example. 
Example 23. Consider $(n, h)=(2,3)$. Then the limit is a point of multiplicity 3 . On the other hand $\operatorname{deg} Z_{1}=9$ while a 3 -ple point has degree 6 , therefore the limit is not the 3 -ple point. There are 3 more linear conditions the liner system has to satisfy. To understand them observe that a plane cubic with 3 double points in general position is a union of 3 lines. These lines intersect the divisor $R$ providing the further linear condition the limit has to satisfy. In particular the linear system $\mathcal{L}_{R}$ is not complete.

Lemma 24. The linear system $\mathcal{L}_{P}$ is complete.

Proof. Consider the exact sequence

$$
0 \rightarrow \mathcal{L}(-P) \rightarrow \mathcal{L} \rightarrow \mathcal{L}_{P} \rightarrow 0
$$

To prove the claim it is enough to show that $\mathrm{h}^{1}(\mathcal{L}(-P))=0$.

The sheaf $\mathcal{L}(-P) \sim \mathcal{O}\left(\sum_{i} 2 \mathcal{E}_{i}-(\mu+1) P\right)$ is the pull-back of the ideal sheaf $\cup_{i} \mathcal{I}_{\sigma_{i}(\Delta)}^{2} \cup \mathbf{m}_{p}^{\mu+1}$ on $\mathbb{A}^{n} \times \Delta$. Hence we have

$$
H^{1}(\mathcal{L}(-P))=H^{1}\left(\cup_{i} \mathcal{I}_{\sigma_{i}(\Delta)}^{2} \cup \mathbf{m}_{p}^{\mu+1}\right)=0 .
$$

The last technical result we need is the following.

Lemma 25. Let $A=\left\{a_{1}, \ldots, a_{l}\right\}$ be a set of $l$ general points in $\mathbb{P}^{n}, n \geq 2$, and $R$ a hyperplane such that $A \cap R=\varnothing$. Let

$$
B=\left\{\left\langle a_{i}, a_{j}\right\rangle \cap R\right\}_{i, j \in\{1, \ldots, l\}} .
$$

Assume that $l=n+1$, then $\mathcal{L}_{n-1,2}(B)$ is nonspecial, that is the points of $B$ impose independent conditions to quadrics.

Proof. $B$ is a set of $\left(\begin{array}{c}n+1 \\ 2\end{array}\right)$ points. Therefore it is enough to prove that there are no quadrics containing $B$. We prove the claim by induction on $n$. For $n=2$ it is easy. Let $\Pi_{i}=\left\langle a_{1}, \ldots, \hat{a_{i}}, \ldots, a_{n+1}\right\rangle \cap R$, then by induction there are no quadrics in $\Pi_{i}$ containing $\Pi_{i} \cap B$. Therefore any quadric containing $B$ has to contain the hyperplanes $\Pi_{i}$ for any $i$. This is enough to conclude since $n+1 \geq 3$.

Remark 26. Note that even if $B$ imposes independent conditions to quadrics, the points of $B$ are not in linear general position. Indeed there are $\left(\begin{array}{c}n+1 \\ t\end{array}\right)$ linear spaces of dimension $t-2$ each containing $\left(\begin{array}{l}t \\ 2\end{array}\right)$ points of $B$. For every choice of $t$ points of $A$, their span is a $\mathbb{P}^{t-1}$, so the corresponding $\left(\begin{array}{l}t \\ 2\end{array}\right)$ points of $B$ lie on a $\mathbb{P}^{t-2}$.

We are ready to compute the limits we need.

Proposition 27. Let $n \geq 2$ and $Z_{1}$ a scheme of $n+1$ double points. Let $\pi$ be a specialization with $n+1$ collapsing points in $\mathbb{A}^{n}$. Let $Z_{0}$ be the flat limit, then $Z_{0}$ is a point of multiplicity 3 together with $\left(\begin{array}{c}n+1 \\ 2\end{array}\right)$ tangent directions.

Proof. There are no quadrics singular along $n+1$ general points of $\mathbb{P}^{n}$ and for $n \geq 2$ the linear system $\mathcal{L}_{n, 3}\left(2^{n+1}\right)$ has non negative dimension, then by Corollary 21, the multiplicity of the limit scheme is 3 . Note that the degree of a 3-ple point is $\left(\begin{array}{c}n+2 \\ 2\end{array}\right)$.

The base locus of cubics in $\mathbb{P}^{n}$ with $(n+1)$ general double points consists of the $\left(\begin{array}{c}n+1 \\ 2\end{array}\right)$ lines joining the points. Each of them cuts a simple point on $R \simeq \mathbb{P}^{n-1}$. By Lemma 25 these points impose independent conditions to cubics. Then by i) in Proposition 4

$$
(n+1)^{2}=\operatorname{deg} Z_{0} \geq\left(\begin{array}{c}
n+2 \\
2
\end{array}\right)+\left(\begin{array}{c}
n+1 \\
2
\end{array}\right)=(n+1)^{2} .
$$


Hence the triple point, together with $\left(\begin{array}{c}n+1 \\ 2\end{array}\right)$ tangent directions, is a 0-dimensional scheme contained in $Z_{0}$ and with the same degree, and we conclude by Proposition 4.

\section{The Induction STEP}

In this section we develop the induction argument we need to prove Theorem 2 , Thanks to Me1 we may assume $n \geq d$. We also assume $d \geq 4$. For cubics a different strategy is needed since linear systems of cubics with a triple point and double points are always special. s Fix a point $q \in \mathbb{P}^{n}$ and a general linear space $\Pi \ni q$, of dimension 3 . Let $Z_{n}$ be a scheme having:

- multiplicity 3 in $q$ together with $\left(\begin{array}{c}n+1 \\ 2\end{array}\right)-s(3, d, n)$ general tangent directions in $q$ and $s(3, d, n)$ tangent directions on $\Pi$

- $k(n, d)-n-2-h(3, d, n)$ general double points, $h(3, d, n)$ general double points on $\Pi$.

We will define the integers $h(3, d, n)$ and $s(3, d, n)$ later on.

At the linear system level let

$$
\mathcal{L}_{n}(d):=\left|\mathcal{O}_{\mathbb{P}^{n}}(d) \otimes \mathcal{I}_{Z_{n}}\right| .
$$

We aim to prove the nonspeciality of $\mathcal{L}_{n}(d)$. For this we degenerate the scheme $Z_{n}$ as follows. Fix a general hyperplane $H \subset \mathbb{P}^{n}$ containing $\Pi$. Let $Z_{n}^{H}$ be a specialization of $Z_{n}$ such that $H$ contains $h(n-1, d, n)$ double points and $s(n-1, d, n)$ tangent directions, at the point $q$. Let $\mathcal{L}_{n}^{H}(d)$ be the specialized linear system.

By Remark 13 the nonspeciality of $\mathcal{L}_{n}(d)$ is implied by the nonspeciality of $\mathcal{L}_{n}^{H}(d)$. For the latter we use the Castelnuovo exact sequence in Remark 15. Therefore we are left to prove that the restricted linear system

$$
\mathcal{L}_{n-1, d}\left(3[s(n-1, d, n)], 2^{h(n-1, d, n)}\right)
$$

and the kernel of the restriction map

$$
\mathcal{L}_{n, d-1}\left(2[s(n, d, n)-s(n-1, d, n)], 2^{k(n, d)-n-2-h(n-1, d, n)}, 1^{h(n-1, d, n)}\right)
$$

are nonspecial, with the points satisfying the prescribed requirements. To prove the nonspeciality of these linear systems we set up an induction argument choosing a sequence of integer in such a way that the first linear system has a unique divisor and then use induction for the latter. Let us introduce the following notation.

Definition 28. Let $s(i, d, n)$ and $h(i, d, n)$ be non-negative integers. Assume $n \geq d$ and $d \geq 4$. Fix a general flag of linear spaces

$$
H_{2} \subset H_{3} \subset H_{4} \subset \ldots \subset H_{n-1} \subset H_{n}=\mathbb{P}^{n},
$$

with $H_{i} \cong \mathbb{P}^{i}, H_{3}=\Pi$ and $q \in H_{2}$. For $i \in\{2, \ldots, n\}$, consider a 0 -dimensional scheme $Z_{i} \subset H_{i}$ such that:

- $Z_{i-1}$ is a flat limit of $Z_{i \mid H_{i-1}}$,

- $Z_{i}$ has multiplicity 3 in $q$ together with $s(i, d, n)$ tangent directions, $s(i-$ $1, d, n)$ of which are tangent directions supported in $H_{i-1}$,

- for every $i \in\{3, \ldots, n\}, Z_{i}$ has $h(i, d, n)$ double points, $h(i-1, d, n)$ of which are supported in $H_{i-1}$.

- $Z_{2}$ has $h(2, d, n)$ double points.

Let

$$
\mathcal{L}_{i}^{H}(d)=\left|\mathcal{O}_{\mathbb{P}^{i}}(d) \otimes \mathcal{I}_{Z_{i}}\right| .
$$

The following is the first step toward proving the induction we need.

Lemma 29. Assume that, for any $n \geq i \geq 2$ there are integers $h(i, d, n) \geq h(i-$ $1, d, n)$ and $s(i, d, n) \geq s(i-1, d, n)$ such that 
i) $\operatorname{expdim} \mathcal{L}_{i}^{H}(d)=i$,

ii) $\operatorname{dim} \mathcal{L}_{i-1}^{H}(d)=i-1$,

iii) $\operatorname{expdim} \mathcal{L}_{i}^{H}(d)-H_{i-1}=0$,

iv) $\operatorname{dim} \mathcal{L}_{i}^{H}(d)-2 H_{i-1} \leq 0$,

v) there is at most one divisor $D \in \mathcal{L}_{i}^{H}(d)$ with $\operatorname{mult}_{q} D>3$,

vi) for $i>3, \mathcal{L}_{i-1}^{H}(d)_{\mid \Pi}=\mathcal{L}_{3, d}\left(3[s(3, d, n)], 2^{h(3, d, n)}\right)$.

vii) $h(i-1, d, n)-h(3, d, n)+s(i, d, n)-s(i-1, d, n)>(i-4)(i+1)$, for $i \geq 5$ and $d \geq 5$,

$h(i-1,4, n)-h(3,4, n)+s(i, 4, n)-s(i-1,4, n)>(i+1)$, for $5 \leq i \leq 8$, $h(i-1,4, n)-h(3,4, n)+s(i, 4, n)-s(i-1,4, n)>(i-7)(i+1)$, for $i \geq 9$.

Then

(1) $\operatorname{dim} \mathcal{L}_{i}^{H}(d)-H_{i-1}=0$,

(2) the divisor in $\mathcal{L}_{i}^{H}(d)-H_{i-1}$ does not contain $\Pi$,

(3) $\mathcal{L}_{i}^{H}(d)$ is nonspecial,

(4) for $i \geq 3, \mathcal{L}_{i}^{H}(d)_{\mid \Pi}=\mathcal{L}_{3, d}\left(3[s(3, d, n)], 2^{h(3, d, n)}\right)$.

Proof. By assumption i) $h(3, d, n)<\frac{\left(\begin{array}{c}d+3 \\ 3\end{array}\right)}{4}-1$, the linear system $\mathcal{L}_{i}^{H}(d)-H_{i-1}$ is

$$
\mathcal{L}_{i, d-1}\left(2[s(i, d, n)-s(i-1, d, n)], 2^{h(i, d, n)-h(i-1, d, n)}, 1^{h(i-1, d, n)}\right),
$$

and it has non negative expected dimension by iii). For $i=4$, using Lemma 16, it is easy to check that the simple points on $\Pi$ impose independent conditions and the general element in $\mathcal{L}_{4}^{H}(d)-\Pi$ does not contain $\Pi$. By vii) and Lemma 18, for $i>4$, the simple base points on $\Pi$ impose independent conditions and the general element in $\mathcal{L}_{i}^{H}(d)-H_{i-1}$ does not contain $\Pi$. This shows (2).

By assumption iv) and Lemma 16. the other simple base points on $H_{i-1}$ impose independent conditions to $\mathcal{L}_{i, d-1}\left(2^{h(i, d, n)-h(i-1, d, n)+1}\right)$.

Similarly assumption v) and Lemma 16 ensure that the tangential directions in $q$ impose independent conditions to $\mathcal{L}_{i, d-1}\left(2^{h(i, d, n)-h(i-1, d, n)+1}\right)$. Therefore we are left to prove that $\mathcal{L}_{i, d-1}\left(2^{h(i, d, n)-h(i-1, d, n)+1}\right)$ is nonspecial. The latter is a linear system with only double points and positive expected dimension. Then by Theorem 7 we know it is nonspecial, keep also in mind Remark 8 . This proves (1).

Then from the Castelnuovo exact sequence and assumption ii) the linear system $\mathcal{L}_{i}^{H}(d)$ is non special. To conclude observe that the nonspeciality of $\mathcal{L}_{i}^{H}(d),(1)$ and vi) yield (4).

To apply Lemma 29 we have first to produce the sequences of integers $h(i, d, n)$ and $s(i, d, n)$.

Proposition 30. Fix integers $n \geq d \geq 4$, and let $i \in\{3, \ldots, n\}$. Assume that the number $k(n, d)$ defined in formula 1 is an integer. Then there are sequences $\{h(i, d, n)\}_{i \in\{2, \ldots, n\}}$ and $\{s(i, d, n)\}_{i \in\{2, \ldots, n\}}$ such that

(1) $\operatorname{expdim} \mathcal{L}_{i, d-1}\left(2[s(i, d, n)-s(i-1, d, n)], 2^{h(i, d, n)-h(i-1, d, n)}, 1^{h(i-1, d, n)}\right)=0$,

(2) $\operatorname{expdim} \mathcal{L}_{i-1, d}\left(3[s(i-1, d, n)], 2^{h(i-1, d, n)}\right)=i-1$,

and the following properties hold:

i) $h(n, d, n)=k(n, d)-1-n-1$,

$$
\begin{gathered}
h(n, d, n)-h(n-1, d, n) \geq \frac{d-1}{(n+2)(n+1)}\left(\begin{array}{c}
n+d \\
n
\end{array}\right)-3>0, \\
h(i+1, d, n)-h(i, d, n) \geq \frac{d-1}{(i+2)(i+1)}\left(\begin{array}{c}
i+d \\
i
\end{array}\right)-2>0
\end{gathered}
$$


for every $i \in\{2, \ldots, n-2\}$ and $h(3, d, n)<\left(\begin{array}{c}d+2 \\ 3\end{array}\right)-4$,

ii) $s(n, d, n)=\left(\begin{array}{c}n+1 \\ 2\end{array}\right)$ and

$$
s(i-1, d, n) \in\left\{\frac{i^{2}-3 i-2}{2}, \ldots, \frac{i^{2}-i-4}{2}\right\},
$$

iii) $s(2, d, n) \geq 0$,

iv) $s(i, d, n) \geq s(i-1, d, n)$ for every $i \in\{4, \ldots, n-1\}$,

v) $s(i, d, n)-s(i-1, d, n)<\left(\begin{array}{c}i+1 \\ 2\end{array}\right)$,

vi) $h(i-1, d, n)-h(3, d, n)+s(i, d, n)-s(i-1, d, n)>(i-4)(i+1)$, for $i \geq 5$ and $d \geq 5$,

$h(i-1,4, n)-h(3,4, n)+s(i, 4, n)-s(i-1,4, n)>(i+1)$, for $5 \leq i \leq 8$, $h(i-1,4, n)-h(3,4, n)+s(i, 4, n)-s(i-1,4, n)>(i-7)(i+1)$, for $i \geq 9$.

Proof. To simplify notation we set for the moment $s_{i}:=s(i, d, n)$, and $h_{i}:=$ $h(i, d, n), k:=k(n, d)$. Set $s_{n}=\left(\begin{array}{c}n+1 \\ 2\end{array}\right)$ and $h_{n}=k-1-n-1$. For $i \in\{3, \ldots, n\}$ define

$$
a_{i}:=: a(i, d):=\left(\begin{array}{c}
i+d-1 \\
i-1
\end{array}\right)-\frac{3 i}{2}-\frac{i^{2}}{2} .
$$
is

The expected dimension of the linear system $\mathcal{L}_{i, d-1}^{H}\left(2\left[s_{i}-s_{i-1}\right], 2^{h_{i}-h_{i-1}}, 1^{h_{i-1}}\right)$

$$
\exp _{i}:=\left(\begin{array}{c}
d-1+i \\
i
\end{array}\right)-1-(i+1)\left(h_{i}-h_{i-1}+1\right)-\left(s_{i}-s_{i-1}\right)-h_{i-1}
$$

Then assumption (1) reads $\exp _{n}=0$ and yields

$$
\begin{aligned}
\left(\begin{array}{c}
d-1+n \\
n
\end{array}\right)-1 & -(n+1)\left(k-n-2-h_{n-1}+1\right)-h_{n-1}-\left(\left(\begin{array}{c}
n+1 \\
2
\end{array}\right)-s_{n-1}\right)=0 \\
n h_{n-1}+s_{n-1} & =-\left(\begin{array}{c}
d-1+n \\
n
\end{array}\right)+2+(n+1) k-(n+1)(n+2)+n+\left(\begin{array}{c}
n+1 \\
2
\end{array}\right) \\
= & -\left(\begin{array}{c}
d-1+n \\
n
\end{array}\right)+2+\left(\begin{array}{c}
n+d \\
n
\end{array}\right)-(n+1)(n+2)+n+\left(\begin{array}{c}
n+1 \\
2
\end{array}\right) \\
= & \left(\begin{array}{c}
n+d-1 \\
n-1
\end{array}\right)+2-(n+1)(n+2)+n+\left(\begin{array}{c}
n+1 \\
2
\end{array}\right)=a_{n} .
\end{aligned}
$$

Therefore $h_{n-1}$ and $s_{n-1}$ satisfy the following equation

$$
n h_{n-1}+s_{n-1}=a_{n} .
$$

Note that $\frac{n^{2}-n-4}{2}-\frac{n^{2}-3 n-2}{2}=n-1$, therefore there is a unique $t \in\left\{\frac{n^{2}-3 n-2}{2}, \ldots, \frac{n^{2}-n-4}{2}\right\}$ such that $a_{n}-t$ is a multiple of $n$; call $s_{n-1}$ that number and define

$$
h_{n-1}=\frac{a_{n}-s_{n-1}}{n} \text {. }
$$

This also settles (2), for $i=n$, since by construction

$$
\exp \operatorname{dim} \mathcal{L}_{n, d}\left(3[s(n, d, n)], 2^{h(n, d, n)}\right)=n
$$

hence

$$
n=\exp _{n}+\exp \operatorname{dim} \mathcal{L}_{n-1, d}\left(3[s(n-1, d, n)], 2^{h(n-1, d, n)}\right)+1 .
$$

In a similar fashion $\exp _{n-1}=0$ gives

$$
(n-1) h_{n-2}+s_{n-2}=\left(\begin{array}{c}
n+d-2 \\
n-2
\end{array}\right)-\frac{n^{2}}{2}-\frac{n}{2}+1=a_{n-1} .
$$


As before $\frac{n^{2}-3 n-2}{2}-\frac{n^{2}-5 n+2}{2}=n-2$, and there is a unique $t \in\left\{\frac{n^{2}-5 n+2}{2}, \ldots, \frac{n^{2}-3 n-2}{2}\right\}$ such that $a_{n-1}-t$ is a multiple of $n-1$; call $s_{n-2}$ that number, and define

$$
h_{n-2}=\frac{a_{n-1}-s_{n-2}}{n-1} .
$$

We iterate the argument, defining $s_{i-1}$ to be the only natural number in $\left\{\frac{i^{2}-3 i-2}{2}, \ldots, \frac{i^{2}-i-4}{2}\right\}$ such that $i \mid a_{i}-s_{i-1}$. Hence we have

$$
i h_{i-1}+s_{i-1}=a_{i}
$$

and conditions ii) and iv) are satisfied.

Next we check $s(2, d, n) \geq 0$. By defini tion $s(2, d, n) \geq-1$. Assume that $s(2, d, n)=-1$. Then

$$
a(3, d)=\frac{(d+1)(d+2)}{2}-9 \equiv-1(\bmod 3)
$$

so $(d+1)(d+2)=6 t+4 \equiv 1(\bmod 3)$, and this is impossible because 1 is irreducible in $\mathbb{Z}_{3}$. Therefore $s(2, d, n) \geq 0$ for any $d$.

Let us check condition v). Assume first that $i<n$. Then

$$
2(s(i, d, n)-s(i-1, d, n)) \leq(i+1)^{2}-i-5-i^{2}+3 i+2=4 i-2<(i+1) i,
$$

for $i \geq 3$. Assume $i=n$, then

$$
2(s(n, d, n)-s(n-1, d, n)) \leq 2\left(\begin{array}{c}
n+1 \\
2
\end{array}\right)-n^{2}+3 n+2=4 n+2<n(n+1)
$$

for $n \geq 4$.

Next we focus on condition i).

Claim 2. Set $i \geq 2$, then

$$
h_{n}-h_{n-1} \geq \frac{d-1}{(n+2)(n+1)}\left(\begin{array}{c}
n+d \\
n
\end{array}\right)-3>0
$$

and

$$
h_{i+1}-h_{i} \geq \frac{d-1}{(i+2)(i+1)}\left(\begin{array}{c}
i+d \\
i
\end{array}\right)-2>0 \text { for every } i \leq n-2
$$

Proof of the Claim. First assume $i=n-1$. Then we have, by Equation (5),

$$
\begin{aligned}
h_{n}-h_{n-1} & =\frac{\left(\begin{array}{c}
n+d \\
n
\end{array}\right)}{n+1}-n-3-\frac{a_{n}-s_{n-1}}{n} \geq \\
& \geq \frac{\left(\begin{array}{c}
n+d \\
n
\end{array}\right)}{n+1}-\frac{\left(\begin{array}{c}
n+d-1 \\
n-1
\end{array}\right)}{n}-3= \\
& \left.=\frac{1}{n(n+1)}\left[\begin{array}{c}
n+d \\
n
\end{array}\right)-(n+1)\left(\begin{array}{c}
n+d-1 \\
n-1
\end{array}\right)\right]-3= \\
& =\frac{d-1}{(n+1) n}\left(\begin{array}{c}
n+d-1 \\
n-1
\end{array}\right)-3 .
\end{aligned}
$$


The case $i<n-1$ is similar but a bit more painful, keep in mind Equation (5)

$$
\begin{aligned}
& h_{i+1}-h_{i}=\frac{a_{i+2}-s_{i+1}}{i+2}-\frac{a_{i+1}-s_{i}}{i+1} \\
& \geq \frac{(i+1) a_{i+2}-(i+1) \cdot \frac{(i+2)^{2}-i-6}{2}-(i+2) a_{i+1}+(i+2) \cdot \frac{(i+1)^{2}-3(i+1)-2}{2}}{(i+2)(i+1)} \\
& =\frac{(i+1) a_{i+2}-(i+2) a_{i+1}}{(i+2)(i+1)}-\frac{3 i^{2}+7 i+6}{2(i+2)(i+1)} \\
& =\frac{1}{(i+2)(i+1)}\left[(i+1) a_{i+2}-(i+2) a_{i+1}\right]-\frac{(i+1)(3 i+4)}{2(i+2)(i+1)}-1 \\
& =\frac{1}{(i+2)(i+1)}\left[(i+1)\left(\begin{array}{c}
i+d+1 \\
i+1
\end{array}\right)-(i+2)\left(\begin{array}{c}
i+d \\
i
\end{array}\right)\right]-\frac{1}{2}-\frac{3 i+4}{2(i+2)}-1 \\
& =\frac{1}{(i+2)(i+1)}\left[(i+1)\left(\begin{array}{c}
i+d \\
i+1
\end{array}\right)-\left(\begin{array}{c}
i+d \\
i
\end{array}\right)\right]-\frac{2 i+3}{i+2}-1 \\
& \geq \frac{1}{(i+2)(i+1)}\left[i \frac{(i+d) !}{(i+1) !(d-1) !}+\frac{(i+d) !}{(i+1) !(d-1) !}-\frac{(i+d) !}{i ! d !}\right]-\frac{2 i+4}{i+2}-1 \\
& =\frac{(i+d) !}{i !(i+2)(i+1)(d-1) !}\left[\frac{i}{i+1}+\frac{1}{i+1}-\frac{1}{d}\right]-3 \\
& =\frac{(i+d) !(d-1)}{i ! d !(i+2)(i+1)}-3 \\
& =\frac{d-1}{(i+2)(i+1)}\left(\begin{array}{c}
i+d \\
i
\end{array}\right)-3 \text {, }
\end{aligned}
$$

for every $i \geq 2$. Note that $h_{i+1}-h_{i}$ increases as $d$ does. For $d=4$ we have

$$
h(i+1,4, n)-h(i, 4, n) \geq \frac{3}{(i+2)(i+1)}\left(\begin{array}{c}
i+4 \\
4
\end{array}\right)-3=\frac{i^{2}+7 i-12}{8}>0,
$$

for $i \geq 2$.

The Claim proves i). We are left with vi). Assume first $i \geq 5$ and $d \geq 5$.

$$
\begin{aligned}
h(i-1, d, n) & -h(3, d, n)+s(i, d, n)-s(i-1, d, n)-(i-4)(i+1) \\
& \geq h(i-1, d, n)-h(3, d, n)-(i-4)(i+1) \\
& =\frac{a(i, d)-s(i-1, d, n)}{i}-\frac{a(4, d)-s(3, d, n)}{4}-(i-4)(i+1) \\
& \geq \frac{\left(\begin{array}{c}
i+d-1 \\
i-1
\end{array}\right)-\frac{3 i}{2}-\frac{i^{2}}{2}-\frac{i^{2}-i-4}{2}}{i}-\frac{\left(\begin{array}{c}
d+3 \\
3
\end{array}\right)-14-1}{4}-(i-4)(i+1) .
\end{aligned}
$$

The latter increases as $d$ does, so

$$
\begin{aligned}
& \frac{\left(\begin{array}{c}
i+d-1 \\
i-1
\end{array}\right)-\frac{3 i}{2}-\frac{i^{2}}{2}-\frac{i^{2}-i-4}{2}}{i}-\frac{\left(\begin{array}{c}
d+3 \\
3
\end{array}\right)-14-1}{4}-(i-4)(i+1) \\
\geq & \frac{\left(\begin{array}{c}
i+4 \\
5
\end{array}\right)-\frac{3 i}{2}-\frac{i^{2}}{2}-\frac{i^{2}-i-4}{2}}{i}-\frac{\left(\begin{array}{l}
8 \\
3
\end{array}\right)-14-1}{4}-(i-4)(i+1) \\
= & \frac{i^{5}+10 i^{4}-85 i^{3}+290 i^{2}-846 i+240}{120 i}>0
\end{aligned}
$$


for every $i \geq 5$. Now assume $d=4$. For $5 \leq i \leq 8$ we have

$$
\begin{aligned}
h(i-1,4, n) & -h(3,4, n)+s(i, 4, n)-s(i-1,4, n)-i-1 \\
& =h(i-1,4, n)+s(i, 4, n)-s(i-1,4, n)-i-6 \\
& \geq h(i-1,4, n)-i-6 \\
& =\frac{a(i, 4)-s(i-1,4, n)}{i}-i-6 \\
& \geq \frac{\left(\begin{array}{c}
i+3 \\
4
\end{array}\right)-\frac{3 i}{2}-\frac{i^{2}}{2}-\frac{i^{2}-i-4}{2}}{i}-i-6 \\
& =\frac{i^{4}+6 i^{3}-37 i^{2}-162 i+48}{24 i}>0
\end{aligned}
$$

for $i \geq 6$. For $i=5$ we have

$$
h(4,4, n)-h(3,4, n)+s(5,4, n)-s(4,4, n)-6=9-5+9-5-6>0 .
$$

Assume now $i \geq 9$.

$$
\begin{aligned}
h(i-1,4, n) & -h(3,4, n)+s(i, 4, n)-s(i-1,4, n)-(i+1)(i-7) \\
& \geq \frac{a(i, 4)-s(i-1,4, n)}{i}-5-(i+1)(i-7) \\
& \geq \frac{\left(\begin{array}{c}
i+3 \\
4
\end{array}\right)-\frac{3 i}{2}-\frac{i^{2}}{2}-\frac{i^{2}-i-4}{2}}{i}-5-(i+1)(i-7) \\
& =\frac{i^{4}-18 i^{3}+131 i^{2}+30 i+48}{24 i}>0
\end{aligned}
$$

for every $i \geq 9$.

Remark 31. There is an interesting consequence of Proposition 30. The sequences $h(i, d, n)$ and $s(i, d, n)$ do not vary with $n$, as long as $i<n$. This is crucial for all the computations we are going to do and opens also interesting generalization of our arguments that we will explore in the future.

Example 32. Here we present the computation in a specific case. Assume $n=5$ and $d=4$. By definition $k(5,4)=21 \in \mathbb{N}$. Following the proof of Proposition 30 , we set $s(5,4,5)=\left(\begin{array}{l}5 \\ 2\end{array}\right)=10$ and $h(5,4,5)=21-2-5=14$. Next we compute

$$
\begin{aligned}
& a(5,4)=\left(\begin{array}{l}
8 \\
4
\end{array}\right)-\frac{15}{2}-\frac{25}{2}=50 \\
& a(4,4)=\left(\begin{array}{l}
7 \\
3
\end{array}\right)-6-8=21 \text { and } \\
& a(3,4)=\left(\begin{array}{l}
6 \\
2
\end{array}\right)-\frac{9}{2}-\frac{9}{2}=6 .
\end{aligned}
$$

Now $s(4,5,4)$ is the only natural number $t \in\{4, \ldots, 8\}$ such that $5 \mid 50-t$. This means $s(4,5,4)=5$ and therefore $h(4,5,4)=\frac{50-5}{5}=9$. In the same way $s(3,5,4)$ is the only number $t \in\{1, \ldots, 4\}$ such that $4 \mid 21-t$. Again this implies $s(3,5,4)=1$ and $h(3,5,4)=\frac{21-1}{4}=5$. Finally $s(2,5,4)$ is the only number $t \in\{0,1\}$ such that $3 \mid 6-t$, so we conclude that $s(2,5,4)=0$ and $h(2,5,4)=3$.

From now on we fix the sequences of integers $h(i, d, n)$ and $s(i, d, n)$ of Proposition 30. The following proves that Lemma 29] assumption iv) is satisfied for this choice of integers.

Lemma 33. Assume $n \geq d \geq 4$ and $(n, d) \neq(4,4)$. Then $\mathcal{L}_{i}^{H}(d)-2 H_{i-1}$ is empty for every $i \in\{3, \ldots, n\}$. 
Proof. By definition $\mathcal{L}_{i}^{H}(d)-2 H_{i-1}$ is

$$
\mathcal{L}_{i, d-2}\left(1[s(i, d, n)-s(i-1, d, n)], 2^{h(i, d, n)-h(i-1, d, n)}\right) .
$$

First assume $d=4, n \geq 5$. Consider $i=3$. A direct computation shows $h(3,4, n)=5, h(2,4,4)=2$, therefore $h(3,4, n)-h(2,4, n) \geq 3=i$. Consider $i \in\{4, \ldots, n-1\}$. By i) in Proposition 30 we have

$$
h(i, 4, n)-h(i-1,4, n) \geq \frac{3}{(i+1) i}\left(\begin{array}{c}
i+3 \\
4
\end{array}\right)-2=\frac{i^{2}+5 i-10}{8} \geq i .
$$

Consider $i=n>d=4$. By i) in Proposition 30 we have

$$
h(n, 4, n)-h(n-1,4, n) \geq \frac{3}{(n+1) n}\left(\begin{array}{c}
n+3 \\
4
\end{array}\right)-3=\frac{n^{2}+5 n-18}{8} \geq n
$$

for $n \geq 6$. If $n=5$ we compute $h(5,4,5)-h(4,4,5)=14-9=5=i$. In all these cases the linear system we are interested in is contained in $\mathcal{L}_{n, 2}\left(1,2^{n}\right)$ which is empty. Next we consider exceptions in Theorem 7 with $d \geq 4$. In our notation these are the cases $(i, d)=(3,6),(4,6),(4,5)$. A direct computation shows that $h(i, d, n)-h(i-1, d, n)$ is greater than the exceptional value of Theorem 7.

Assume then $n \geq d>4$. By hypothesis the linear system $\mathcal{L}_{i, d-2}\left(2^{h(i, d, n)-h(i-1, d, n)}\right)$ is nonspecial. Then by i) in Proposition 30, for $i<n$ we have

$$
\begin{aligned}
\operatorname{virtdim} \mathcal{L}_{i, d-2}( & \left.1[s(i, d, n)-s(i-1, d, n)], 2^{h(i, d, n)-h(i-1, d, n)}\right) \\
& <\left(\begin{array}{c}
d+i-2 \\
i
\end{array}\right)-(i+1)(h(i, d, n)-h(i-1, d, n)) \\
& \leq\left(\begin{array}{c}
d+i-2 \\
i
\end{array}\right)-(i+1)\left[\frac{d-1}{(i+1) i}\left(\begin{array}{c}
i+d-1 \\
i-1
\end{array}\right)-2\right] \\
& =\frac{(d+i-2) !}{i !(d-2) !}-\frac{d-1}{i} \cdot \frac{(i+d-1) !}{(i-1) ! d !}+2(i+1) \\
& =\frac{(i+d-2) !}{i !(d-2) !}-(d-1) \cdot \frac{(i+d-1) !}{i ! d !}+2(i+1) \\
& =\frac{(i+d-2) !}{i !(d-2) !}\left[1-(d-1) \cdot \frac{i+d-1}{d(d-1)}\right]+2(i+1) \\
& =\left(\begin{array}{c}
i+d-2 \\
d-2
\end{array}\right) \cdot \frac{1-i}{d}+2(i+1) .
\end{aligned}
$$

Note that the latter decreases as $d$ increases. For $d=5$ we get

$$
\frac{1-i}{5}\left(\begin{array}{c}
i+3 \\
3
\end{array}\right)+2(i+1) \leq \frac{-i^{4}-5 i^{3}-5 i^{2}+65 i+66}{30} \leq 0
$$

for every $i \geq 3$.

To conclude assume $i=n$. Then the virtual dimension is bounded as follows

$$
\begin{gathered}
\operatorname{virtdim} \mathcal{L}_{n, d-2}\left(1[s(n, d, n)-s(n-1, d, n)], 2^{h(n, d, n)-h(n-1, d, n)}\right) \\
<\frac{1-n}{d}\left(\begin{array}{c}
n+d-2 \\
d-2
\end{array}\right)+3(n+1) .
\end{gathered}
$$

As before it decreases as $d$ increase. For $d=5$ we have

$$
\frac{1-n}{5}\left(\begin{array}{c}
n+3 \\
3
\end{array}\right)+3(n+1)=\frac{-n^{4}-5 n^{3}-5 n^{2}+95 n+96}{30}<0,
$$

for every $n \geq 5$.

We are in the condition to state and prove the induction step we described. 
Proposition 34. Assume $n \geq d \geq 4$ and $(n, d) \neq(4,4)$. Let $i \in\{3, \ldots, n\}$. Suppose that

a) $\mathcal{L}_{i-1}^{H}(d)$ is nonspecial

b) there is at most one divisor $D \in \mathcal{L}_{i-1}^{H}(d)$ with $\operatorname{mult}_{q} D>3$

then $\mathcal{L}_{i}^{H}(d)$ is nonspecial and there is at most one divisor $D \in \mathcal{L}_{i}^{H}(d)$ with $\operatorname{mult}_{q} D>$ 3 .

Proof. Recall $q \in H_{i-1}$. First we check that the conditions i), ii), iii), iv), vii) in Lemma 29 are satisfied. Point i) is (2) in Proposition 30, ii) is a) and (2) in Proposition [30, iii) is (1) in Proposition [30, iv) is Lemma 33, and vii) is vi) in Proposition 30 .

We are left to prove that there is at most one divisor $D \in \mathcal{L}_{i}^{H}(d)$ with $\operatorname{mult}_{q} D>$ 3. All divisors $D \in \mathcal{L}_{i}^{H}(d)$ with mult $_{q} D>3$ either contain $H_{i-1}$ or restricts to divisors $D_{\mid H_{i-1}} \in \mathcal{L}_{i-1}^{H}(d)$ with mult $_{q} D_{\mid H_{i-1}}>3$. On the other hand, by assumption b), if there is a pencil of these divisors the unique divisor in $\mathcal{L}_{i}^{H}(d)-$ $H_{i-1}$ has multiplicity at least 4 in $q$. Therefore to conclude it is enough to prove that $\operatorname{mult}_{q} D=3$ for the divisor $D$ with $D \supset H_{i-1}$. The divisor $D-H_{i-1}$ is in

$$
\mathcal{L}_{i, d-1}\left(2[s(i, d, n)-s(i-1, d, n)], 2^{h(i, d, n)-h(i-1, d, n)}, 1^{h(i-1, d, n)}\right) .
$$

A straightforward computation shows that

$$
h(i, d, n)-h(i-1, d, n)<\left\lceil\frac{\left(\begin{array}{c}
i+d-1 \\
i
\end{array}\right)}{i+1}\right\rceil-i-1,
$$

therefore by Proposition 17 the linear system $\mathcal{L}_{i, d-1}\left(3,2^{h(i, d, n)-h(i-1, d, n)}\right)$ is nonspecial. By Lemma $33 \mathcal{L}_{i, d-1}\left(2[s(i, d, n)-s(i-1, d, n)], 2^{h(i, d, n)-h(i-1, d, n)}\right)-H_{i-1}$ is empty and so is $\mathcal{L}_{i, d-1}\left(3,2^{h(i, d, n)-h(i-1, d, n)}\right)-H_{i-1}$.

Hence, arguing as in Proposition 29, we use Lemma 18 and Lemma 16 to ensure that the simple points on the hyperplane impose independent conditions and the linear system $\mathcal{L}_{i, d-1}\left(3,2^{h(i, d, n)-h(i-1, d, n)}, 1^{h(i-1, d, n)}\right)$ is nonspecial. Point (1) in Proposition 30 gives

$$
\operatorname{expdim} \mathcal{L}_{i, d-1}\left(2[s(i, d, n)-s(i-1, d, n)], 2^{h(i, d, n)-h(i-1, d, n)}, 1^{h(i-1, d, n)}\right)=0 .
$$

Point v) in Proposition 30 gives $s(i, d, n)-s(i-1, d, n)<\left(\begin{array}{c}i+1 \\ 2\end{array}\right)$, then

$$
\operatorname{virtdim} \mathcal{L}_{i, d-1}\left(3,2^{h(i, d, n)-h(i-1, d, n)}, 1^{h(i-1, d, n)}\right)<
$$

$<\operatorname{virtdim} \mathcal{L}_{i, d-1}\left(2[s(i, d, n)-s(i-1, d, n)], 2^{h(i, d, n)-h(i-1, d, n)}, 1^{h(i-1, d, n)}\right)=0$.

Since $\mathcal{L}_{i, d-1}\left(3,2^{h(i, d, n)-h(i-1, d, n)}, 1^{h(i-1, d, n)}\right)$ is nonspecial it is empty and any divisor in $\mathcal{L}_{i, d-1}\left(2[s(i, d, n)-s(i-1, d, n)], 2^{h(i, d, n)-h(i-1, d, n)}, 1^{h(i-1, d, n)}\right)$ has a double point in $q$.

The next proposition proves the first step of our induction argument.

Proposition 35. Assume $n \geq d \geq 4$ and $(n, d) \neq(4,4)$. Then the linear system $\mathcal{L}_{2, d}\left(3[s(2, d, n)], 2^{h(2, d, n)}\right)$ is nonspecial and there is at most one divisor $D \in$ $\mathcal{L}_{2, d}\left(3[s(2, d, n)], 2^{h(2, d, n)}\right)$ with mult $_{q} D>3$.

Proof. A simple check of the list in [CM, Theorem 7.1] shows that the linear systems $\mathcal{L}_{2, d}\left(3,2^{h(2, d, n)}\right)$ and $\mathcal{L}_{2, d}\left(4,2^{h(2, d, n)}\right)$ are nonspecial for $d \geq 5$. While a direct computation shows that $\mathcal{L}_{2,4}\left(3,2^{h(2,4, n)}\right)$ is nonspecial and $\operatorname{dim} \mathcal{L}_{2,4}\left(4,2^{h(2,4, n)}\right)=$ 0 . In particular $\mathcal{L}_{2, d}\left(4,2^{h(2, d, n)}\right)$ is empty for $d \geq 5$ and has dimension 0 for $d=4$.

We are left to study the $s(2, d, n)$ tangent direction. If $s(2, d, n)=0$, we are done. Suppose $s(2, d, n)=1$. This is possible only for $d \geq 5$. Since $\mathcal{L}_{2, d}\left(4,2^{h(2, d, n)}\right)$ is empty and $\mathcal{L}_{2, d}\left(3,2^{h(2, d, n)}\right)$ is of positive dimension we conclude, by Lemma 16 , 
that $\mathcal{L}_{2, d}\left(3[s(2, d, n)], 2^{h(2, d, n)}\right)$ is nonspecial. Moreover for $d \geq 5$ any divisor in $\mathcal{L}_{2, d}\left(3,2^{h(2, d, n)}\right)$ has multiplicity 3 in $q$, while there is a unique divisor with multiplicity 4 in $\mathcal{L}_{2,4}\left(3,2^{h(2,4, n)}\right)$.

We are ready to state the nonspeciality result we were looking for.

Proposition 36. If $n \geq d \geq 4$ and $(n, d) \neq(4,4)$, then

i) the linear system $\mathcal{L}_{n}^{H}(d)$ and $\mathcal{L}_{n}(d)$ are nonspecial,

ii) there is at most one divisor $D \in \mathcal{L}_{n}^{H}(d)$ with $\operatorname{mult}_{q} D>3$.

Proof. By Proposition $35 \mathcal{L}_{2, d}\left(3[s(2, d, n)], 2^{h(2, d, n)}\right)$ satisfies i) and ii). Then by Proposition $34 \mathcal{L}_{n}^{H}(d)$ satisfies i) and ii). We already observed that $\mathcal{L}_{n}(d)$ is nonspecial if $\mathcal{L}_{n}^{H}(d)$ is nonspecial.

\section{The genus Bound}

In this section we aim to bound from below the sectional genus of linear systems $\mathcal{L}_{n}^{H}(d)$. We start from $\mathcal{L}_{2, d}\left(3[s(2, d, n)], 2^{h(2, d, n)}\right)$.

Proposition 37. The sectional genus of $\mathcal{L}_{2, d}\left(3[s(2, d, n)], 2^{h(2, d, n)}\right)$ is 0 for $d=4,5$ and it is positive for $d \geq 6$.

Proof. The general element in $\mathcal{L}_{2, d}\left(3[s(2, d, n)], 2^{h(2, d, n)}\right)$ has a triple point in $q$, by Proposition [35, and double points at the rest of the assigned points by [CM], Theorem 8.1].

Claim 3. If $d \geq 8$ the general element $D \in \mathcal{L}_{2, d}\left(3[s(2, d, n)], 2^{h(2, d, n)}\right)$ is irreducible.

Proof of the Claim. Assume that the general element $D \in \mathcal{L}_{2, d}\left(3[s(2, d, n)], 2^{h(2, d, n)}\right)$ is reducible. Then $D=D_{1}+D_{2}$, set

$$
d-1 \geq b_{i}=\operatorname{deg} D_{i}>0
$$

and, by monodromy, $m_{i}:=$ mult $_{p_{j}} D_{i}$. Then $m_{1}+m_{2}=2$ so, up to order, there are two possibilities: either $m_{1}=0$ and $m_{2}=2$, or $m_{1}=m_{2}=1$.

Assume first that $m_{2}=2$, that is we are assuming that $\mathcal{L}_{2, b_{2}}\left(2^{h(2, d, n)}\right)$ is not empty. We are assuming $d \geq 8$, then $h(2, d, n) \geq 12$. Therefore, by Theorem 7 this linear system is nonspecial and its virtual dimension is

$$
\left(\begin{array}{c}
b_{2}+2 \\
2
\end{array}\right)-3 h(2, d, n)-1=\left(\begin{array}{c}
b_{2}+2 \\
2
\end{array}\right)-a(3, d)+s(2, d, n)-1 .
$$

A straightforward computation shows that this is non negative only for $d<8$.

Assume $m_{1}=m_{2}=1$. Then there are plane curves $D_{i}$ of degree $b_{i}$ through $p_{1}, \ldots, p_{h(2, d, n)}$, that is $D_{i} \in \mathcal{L}_{2, b_{i}}\left(1^{h(2, d, n)}\right)$. We may assume $b_{1} \leq b_{2}$, then the virtual dimension of $\mathcal{L}_{2, b_{i}}\left(1^{h(2, d, n)}\right)$ is

$$
\left(\begin{array}{c}
b_{i}+2 \\
2
\end{array}\right)-h(2, d, n)-1
$$

and this is non negative only if

$$
3 b_{i}^{2}+9 b_{i} \geq d^{2}+3 d-22 .
$$

Therefore

$$
\begin{gathered}
3 b_{1}^{2}+9 b_{1} \geq b_{1}^{2}+b_{2}^{2}+2 b_{1} b_{2}+3 b_{1}+3 b_{2}-22 \\
2 b_{1}^{2}+6 b_{1} \geq b_{2}^{2}+2 b_{1} b_{2}+3 b_{2}-22 \geq b_{1}^{2}+2 b_{1}^{2}+3 b_{1}-22 \\
b_{1}^{2}-3 b_{1}-22 \leq 0
\end{gathered}
$$

so $b_{1} \leq 6$. But then by (6) we have

$$
2 b_{1}^{2}+6 b_{1} \geq b_{2}^{2}+2 b_{1} b_{2}+3 b_{2}-22 \geq b_{2}^{2}+2 b_{1}^{2}+3 b_{2}-22
$$




$$
\begin{gathered}
6 b_{1} \geq b_{2}^{2}+3 b_{2}-22 \geq b_{2}^{2}+3 b_{1}-22 \\
3 b_{1} \geq b_{2}^{2}-22 \\
b_{2}^{2} \leq 3 b_{1}+22 \leq 18+22=40
\end{gathered}
$$

and we conclude $b_{2} \leq 6$.

Since $h(2,8, n)=12$ we have $b_{i} \geq 4$. Therefore the only possibilities are $b_{1}=4$ and $10 \geq d \geq 8, b_{1}=5$ and $11 \geq d \geq 10$ or $b_{1}=6$ and $d=12$. A simple computation gives: $h(2,8, n)=12, h(2,9, n)=15, h(2,10, n)=19, h(2,11, n)=23$ and $h(2,12, n)=27$. The divisor $D$ has a triple point at $q$. Therefore the divisor $D_{1}$ has to belong to one of the following linear systems:

$$
\begin{aligned}
d=8 & \mathcal{L}_{2,4}\left(2,1^{12}\right), \\
d=9 & \mathcal{L}_{2,4}\left(1^{15}\right), \\
d=10 & \text { either } \mathcal{L}_{2,4}\left(1^{19}\right) \text { or } \mathcal{L}_{2,5}\left(2,1^{19}\right), \\
d=11 & \mathcal{L}_{2,5}\left(1^{23}\right), \\
d=12 & \mathcal{L}_{2,6}\left(2,1^{27}\right) .
\end{aligned}
$$

An easy check shows that they are all empty.

Set $d \geq 8$. Since the general element is irreducible the map $\varphi_{2, d}$ is dominant. Assume that the geometric genus of $D$ is 0 and, beside the singularities we imposed, there are $l$ singular points of multiplicity $m_{i}$ and $t$ simple base points. Then we get

$$
0=\frac{(d-1)(d-2)}{2}-3-h(2, d, n)-\sum_{i=1}^{l} \frac{m_{i}\left(m_{i}-1\right)}{2}
$$

and

$$
1 \leq d^{2}-9-4 h(2, d, n)-s(2, d, n)-t-\sum_{i=1}^{l} m_{i}^{2}
$$

This yields

$0 \leq d^{2}-10-4 h(2, d, n)-s(2, d, n)-t-\sum_{i=1}^{l} m_{i}^{2}-\left(\frac{(d-1)(d-2)}{2}-3-h(2, d, n)-\sum_{i=1}^{l} \frac{m_{i}\left(m_{i}-1\right)}{2}\right)$

and, recall Equation (5),

$$
\begin{aligned}
0 & \leq \frac{d^{2}+3 d}{2}-8-3 h(2, d, n)-s(2, d, n)-t-\sum_{i=1}^{l} \frac{m_{i}\left(m_{i}+1\right)}{2} \\
& =\frac{d^{2}+3 d}{2}-8-a(2, d)-t-\sum_{i=1}^{l} \frac{m_{i}\left(m_{i}+1\right)}{2} \\
& =-t-\sum_{i=1}^{l} \frac{m_{i}\left(m_{i}+1\right)}{2},
\end{aligned}
$$

therefore $l=0$ and $t=0$.

To conclude we compute the genus

$$
\begin{aligned}
0 & =2 g=(d-1)(d-2)-6-2 h(2, d, n)=d^{2}-3 d-4-2 h(2, d, n) \\
& \geq d^{2}-3 d-4-\frac{2}{3} a(2, d)=\frac{2}{3}\left(d^{2}-6 d+2\right) .
\end{aligned}
$$

This contradicts the assumption $d \geq 8$.

Assume $d=7$, then $h(2,7, n)=9, s(2,7, n)=0$. Let $C_{1} \in \mathcal{L}_{2,3}\left(1,1^{8}\right)$ be a cubic through $q$ and $\left\{p_{1}, \ldots, p_{8}\right\}$, and $C_{2} \in \mathcal{L}_{2,4}\left(2,1^{7}, 2\right)$, a quartic singular in $q$ and $p_{9}$ and passing through $\left\{p_{1}, \ldots, p_{8}\right\}$. Then $D=C_{1}+C_{2}$ is a reducible element of positive genus in $\mathcal{L}_{2,7}\left(3,2^{9}\right)$ and we conclude by Remark 11 , 
Assume $d=6$, then $h(2,6, n)=6, s(2,6, n)=1$. Let $C_{1} \in \mathcal{L}_{2,3}\left(2,1^{6}\right)$ and $C_{2} \in$ $\mathcal{L}_{2,3}\left(1[1], 1^{6}\right)$ be curves. Then $D=C_{1}+C_{2}$ is a reducible element in $\mathcal{L}_{2,6}\left(3[1], 2^{6}\right)$. The curve $C_{2}$ is not rational therefore the sectional genus of $\mathcal{L}_{2,6}\left(3[1], 2^{6}\right)$ is positive by Remark 11 .

For $d=4$ and $d=5$ it is an easy computation to see that the movable part of $\mathcal{L}_{2, d}\left(3[s(2, d, n)], 2^{h(2, d, n)}\right)$ is given respectively by $\mathcal{L}_{2,2}\left(1^{3}\right)$ and $\mathcal{L}_{2,3}\left(2,1^{4}\right)$, which have genus 0 .

For $d=4,5$ we bound the genus of $\mathcal{L}_{3, d}\left(3[s(3, d, n)], 2^{h(3, d, n)}\right)$.

Proposition 38. The sectional genus of $\mathcal{L}_{3,5}\left(3[s(3,5, n)], 2^{h(3,5, n)}\right)$ is positive, for $n \geq 4$.

Proof. A direct computation gives $s(3,5, n)=2, h(3,5, n)=10, s(2,5, n)=0$, and $h(2,5, n)=4$. Let $H+S \in \mathcal{L}_{3}^{H}(5)$ be the unique divisor containing $H$. By Remark 11] it is enough to prove that $\mathcal{L}_{3,5}\left(3[s(3,5, n)], 2^{h(3,5, n)}\right)_{\mid S}$ has positive sectional genus.

The surface

$$
S \in \mathcal{L}_{3,4}\left(2[2], 2^{6}, 1^{4}\right)\left(q\left[t_{1}, t_{2}\right], p_{1}, \ldots, p_{6}, z_{1}, \ldots, z_{4}\right)
$$

is a quartic surface, the $p_{i}$ 's are general and the $z_{i}$ are general on $H$. By $\mathrm{Me} 1$, Theorem 4.1], $\mathcal{L}_{3,4}\left(2^{7}\right)$ is nonspecial and the general element has 7 ordinary double points as unique singularities. The $z_{i}$ 's are in general position on $H$ therefore the general element in

$$
\mathcal{L}_{3,4}\left(2^{7}, 1^{4}\right)\left(q, p_{1}, \ldots, p_{6}, z_{1}, \ldots, z_{4}\right)
$$

has only 7 ordinary double points. The linear system $\mathcal{L}_{3,4}\left(3,2^{5}\right)\left(q, p_{1}, \ldots, p_{5}\right)$ is nonspecial of dimension 4 by Proposition 17 and $\mathcal{L}_{3,3}\left(2,2^{5}\right)$ is empty therefore

$$
\mathcal{L}_{3,4}\left(3,2^{5}, 1^{5}\right)\left(q, p_{1}, \ldots, p_{5}, p_{6}, z_{1}, \ldots, z_{4}\right)
$$

and henceforth $\mathcal{L}_{3,4}\left(3,2^{6}, 1^{4}\right)$ are empty. This shows that for a general choice of 2 tangent directions the surface

$$
S \in \mathcal{L}_{3,4}\left(2[2], 2^{6}, 1^{4}\right)\left(q\left[t_{1}, t_{2}\right], p_{1}, \ldots, p_{6}, z_{1}, \ldots, z_{4}\right)
$$

has 7 ordinary double points as unique singularities. In particular $S$ is a, singular, $K 3$ surface and it is not uniruled. Therefore $\mathcal{L}_{3,5}\left(3[s(3,5, n)], 2^{h(3,5, n)}\right)_{\mid S}$ has positive sectional genus.

Proposition 39. The sectional genus of $\mathcal{L}_{3,4}\left(3[s(3,4, n)], 2^{h(3,4, n)}\right)$ is positive, for $n \geq 4$.

Proof. Our choice of integers is $s(3,4, n)=1, h(3,4, n)=5, s(2,4, n)=0$, and $h(2,4, n)=2$. Let $S+H$ be the unique element in $\mathcal{L}_{3}^{H}(4)$ containing the hyperplane $H$. Then

$$
S \in \mathcal{L}_{3,3}\left(2[1], 2^{3}, 1^{2}\right)\left(q[t], p_{1}, p_{2}, p_{3}, p_{4}, p_{5}\right)
$$

is a cubic surface with 4 double points. It is easy to prove, with reducible elements, that the scheme base locus of $\mathcal{L}_{3}^{H}(4)$ is given by the assigned singularities and the lines spanned by $q$ and $\left\{p_{1}, p_{2}, p_{3}\right\}$. Hence the fixed component of $\mathcal{L}_{3}^{H}(4)_{\mid S}$ is given by the 3 lines spanned by $q$ and $p_{1}, p_{2}$, and $p_{3}$. Then the general element in the movable part of $\mathcal{L}_{3}^{H}(4)_{\mid S}$ has a triple point in $p_{1}$ and a double point in $p_{4}$ and therefore positive genus. This is enough to conclude by Remark 11.

We conclude the section collecting all the results we need.

Proposition 40. The sectional genus of $\mathcal{L}_{3, d}\left(3[s(3, d, n)], 2^{h(3, d, n)}\right)$ is positive for $n, d \geq 4$. 
Proof. For $d=4,5$ this is the content of Propositions [39, 38, For higher degrees observe that by construction a general curve section of $\mathcal{L}_{2, d}\left(3[s(2, d, n)], 2^{h(2, d, n)}\right)$ is an irreducible component of a curve section of $\mathcal{L}_{3}^{H}(d)$ and therefore the sectional genus of $\mathcal{L}_{3, d}\left(3[s(3, d, n)], 2^{h(3, d, n)}\right)$ is bounded by the sectional genus of $\mathcal{L}_{2, d}\left(3[s(2, d, n)], 2^{h(2, d, n)}\right)$. Thus we conclude by Proposition 37

\section{Cubics and proof of Theorem 2}

Fix a pair $(n, d)$, with $n \geq d \geq 4$, a linear space $\Pi \cong \mathbb{P}^{3}$ and a point $q \in \Pi$. Let $Z_{0}$ be the 0 -dimensional scheme obtained as a limit of $k(n, d)-1$ double points $n+1$ of which collapse to the point $q$ with $s(3, d, n)$ tangent directions and $h(3, d, n)$ double points on $\Pi$. Such a degeneration always exists since $s(3, d, n) \leq 4$. Let

$$
\mathcal{L}_{0}(n, d):=\left|\mathcal{O}_{\mathbb{P}}(d) \otimes \mathcal{I}_{Z_{0}}\right|
$$

be the associated linear system.

Lemma 41. If $n \geq d \geq 4$ and $(n, d) \neq(4,4)$, then the linear system $\mathcal{L}_{0}(n, d)$ is non special and its sectional genus is positive.

Proof. Thanks to Proposition 36 for the nonspeciality we have only to worry about the tangent directions. Let $T$ be the set of $\left(\begin{array}{c}n+1 \\ 2\end{array}\right)$ tangent directions. Set $T_{i}:=$ $\left\{y_{1}, \ldots, y_{i}\right\} \subset T$ be a subset of $i$ tangent directions. Assume that $\mathcal{L}_{n, d}\left(3[i], 2^{h}\right)$ is nonspecial and $\mathcal{L}_{n, d}\left(3[i+1], 2^{h}\right)$ is special for a general choice of $h$ double points. Let $\varphi$ be the map associated to the linear system $\mathcal{L}_{n, d}\left(3[i], 2^{h-1}\right)$.

Since $T$ imposes independent conditions on cubics we may assume that $y_{j} \notin$ $\operatorname{Bs} \mathcal{L}_{n, d}\left(3[i], 2^{h-1}\right)$, for $j>i$. The speciality of $\mathcal{L}_{n, d}\left(3[i+1], 2^{h}\right)$ forces $\varphi\left(y_{j}\right)$ to be a vertex of $\varphi\left(\mathbb{P}^{n}\right)$, for $j>i$. Hence a general divisor $D \in \mathcal{L}_{n, d}\left(3[i+1], 2^{h}\right)$ is singular at $y_{j}$, for $j>i$. By a monodromy argument then a general divisor in $\mathcal{L}_{n, d}\left(3\left[\left(\begin{array}{c}n+1 \\ 2\end{array}\right)\right], 2^{h}\right)$ is singular along $T$. Since $\mathcal{L}_{n-1,3}\left(2^{\left(\begin{array}{c}n+1 \\ 2\end{array}\right)}\right)(T)$ is empty this yields

$$
\mathcal{L}_{n, d}\left(3\left[\left(\begin{array}{c}
n+1 \\
2
\end{array}\right)\right], 2^{h}\right) \subseteq \mathcal{L}_{n, d}\left(4,2^{h}\right)
$$

and contradicts Proposition 36 ii) for $h \leq k(n, d)-n-2$.

We are left to bound the sectional genus of $\mathcal{L}_{0}(n, d)$. Let $\tilde{T}_{i}$ be a set of $\left(\begin{array}{c}n+1 \\ 2\end{array}\right)-i$ general tangent directions. Let

$$
\mathcal{L}\left(T_{i}\right):=\mathcal{L}_{n, d}\left(3\left[\left(\begin{array}{c}
n+1 \\
2
\end{array}\right)\right], 2^{k(n, d)-n-2}\right)\left(q\left[T_{i} \cup \tilde{T}_{i}\right], p_{1}, \ldots, p_{k(n, d)-n-2}\right) .
$$

By definition we have $\mathcal{L}\left(T_{1}\right)=\mathcal{L}_{n}^{H}(d)$. Fix $D_{1}, \ldots, D_{n-3} \in \mathcal{L}_{n}^{H}(d)$ general divisors containing $\Pi$ and $Y_{1}, Y_{2} \in \mathcal{L}_{n}^{H}(d)$ general divisors. Then, by Lemma 29 (2), $\Pi$ is an irreducible component of $D_{1} \cdot \ldots \cdot D_{n-3}$ and, by Lemma 29 (4), an irreducible component of $Y_{1} \cdot Y_{2} \cdot D_{1} \cdot \ldots \cdot D_{n-3}$ is a curve section of $\mathcal{L}_{3, d}\left(3[s(3, d, n)], 2^{h(3, d, n)}\right)$. Hence, by Proposition 40 and Remark 11, the claim is true for $i=1$.

To conclude we increase $i$ recursively. Fix $D_{1}, \ldots, D_{n-3} \in \mathcal{L}\left(T_{i+1}\right)$ general divisors containing $\Pi$ and $Y_{1}, Y_{2} \in \mathcal{L}\left(T_{i+1}\right)$ general divisors. By construction $s(3, n, d)>0$ therefore we may assume that $\mathcal{L}\left(T_{i+1}\right)$ is a specialization of $\mathcal{L}\left(T_{i}\right)$ moving a tangent direction in $\Pi$. In this degeneration all sections in $\mathcal{L}\left(T_{i}\right)$ containing $\Pi$ are also sections of $\mathcal{L}\left(T_{i+1}\right)$. This shows that $\Pi$ is an irreducible component of $D_{1} \cdot \ldots \cdot D_{n-3}$. Next we may consider $\mathcal{L}\left(T_{i+1}\right)$ as a specialization of $\mathcal{L}\left(T_{i}\right)$ moving a point outside $\Pi$. Via this degeneration we prove that $\mathcal{L}\left(T_{i+1}\right)_{\mid \Pi}=\mathcal{L}\left(T_{i}\right)_{\mid \Pi}$ and therefore an irreducible component of $Y_{1} \cdot Y_{2} \cdot D_{1} \cdot \ldots \cdot D_{n-3}$ is a curve section of $\mathcal{L}_{3, d}\left(3[s(3, d, n)], 2^{h(3, d, n)}\right)$. Then Proposition 40] and Remark 11] allow to conclude. 
5.1. $(d=3)$. The argument we used for forms of degree $d \geq 4$ does not work for cubics. Linear systems of cubics with a triple point and at least a double point are always special. This forces us to apply a different strategy to study the degree of the map associated to $\mathcal{L}_{n, 3}\left(2^{k}\right)$. This is inspired by BO and Pos2 proof of Alexander-Hirschowitz Theorem. Note that we are interested in integers $n$ such that

$$
k(n):=k(n, 3)=\frac{\left(\begin{array}{c}
n+3 \\
3
\end{array}\right)}{n+1}
$$

is an integer. This is equivalent to say that $n \equiv 0,1(3)$. This property is preserved by codimension 3 linear spaces. This simple observation suggests the following induction procedure.

Assume $k(i)$ is an integer. Let $Z_{1} \subset \mathbb{P}^{i}$ be a 0 -dimensional scheme of $k(i)-1$ general double points. Fix a general codimension 3 linear space $\Pi \subset \mathbb{P}^{i}$ and let $Z_{0}$ be a specialization of $Z_{1}$ with $k(i-3)-1$ double points on $\Pi$. Therefore the linear system $\mathcal{L}_{i, 3}\left(2^{k(i)-1}\right)$ specializes to a linear system $\mathcal{L}_{0}$ and we may split $\mathcal{L}_{0}$ as a direct sum of

$$
\tilde{\mathcal{L}} \text { and } \mathcal{L}_{i-3,3}\left(2^{k(i-3)-1}\right),
$$

where $\tilde{\mathcal{L}}$ is the linear system of cubics containing $\Pi$ and singular in $i+1=k(i)-$ $k(i-3)$ general points of $\mathbb{P}^{i}$ and in $k(i-3)$ general points of $\Pi$. The linear system $\tilde{\mathcal{L}}$ is known to be nonspecial by [BO, Proposition 5.4], see also [Pos2, subsection 5.2], and $\mathcal{L}_{i-3,3}\left(2^{k(i-3)-1}\right)$ is nonspecial by Theorem 7 . Let $g_{i}$ be the sectional genus of $\mathcal{L}_{i, 3}\left(2^{k(i)-1}\right)$, for $i \equiv 0,1(3)$. Let $D_{1}, D_{2}, D_{3}$ be three general cubics containing $\Pi$. Considering the $\mathbb{P}^{3}$ 's spanned by 4 double points, it is easy to check that $\Pi$ is an irreducible component of $D_{1} \cdot D_{2} \cdot D_{3}$, hence

$$
g_{i} \geq g_{i-3}
$$

for $i \geq 6$.

Lemma 42. $g_{6}>0$.

Proof. The number $k(6)$ is 12 . Let $Z_{0}=\left\{p_{1}, \ldots, p_{8}, z_{1}, z_{2}, z_{3}\right\} \subset \mathbb{P}^{6}$ be a specialization with the $p_{i}$ 's on a hyperplane $H$ and $z_{j}$ general. Then $\mathcal{L}_{6,3}\left(2^{11}\right)$ specializes to a linear system $\mathcal{L}_{6,3}^{H}=\mathcal{L}+\mathcal{L}_{5,3}\left(2^{8}, 1^{3}\right)$, with $\operatorname{dim} \mathcal{L}_{6,3}^{H}=6$. It is easy to see that $\operatorname{dim} \mathcal{L}=1$ and $\mathcal{L}=H+\Lambda$ with $\Lambda$ a pencil of quadrics of rank 4 with vertex $\left\langle z_{1}, z_{2}, z_{3}\right\rangle$. Then $M:=\operatorname{Bs} \Lambda$ is a cone over a normal elliptic curve in $\mathbb{P}^{3}$. In particular $M$ is not rationally connected and therefore $\mathcal{L}_{6,3}^{H}$ has positive sectional genus. Then, by Remark 11, we conclude that $g_{6}>0$.

Lemma 43. $g_{7}>0$.

Proof. The number $k(7)$ is 15 . Let $Z_{0}=\left\{p_{1}, \ldots, p_{11}, z_{1}, z_{2}, z_{3}\right\} \subset \mathbb{P}^{7}$ be a specialization with the $p_{i}$ 's on a hyperplane $H$ and $z_{j}$ general. Then $\mathcal{L}_{7,3}\left(2^{14}\right)$ specializes to a linear system $\mathcal{L}_{7,3}^{H}=\mathcal{L}+\mathcal{L}_{6,3}\left(2^{11}, 1^{3}\right)$, with $\operatorname{dim} \mathcal{L}_{7,3}^{H}=7$. It is easy to see that $\operatorname{dim} \mathcal{L}=3$ and $\mathcal{L}=H+\Lambda$ with $\Lambda$ a linear system of quadrics of rank 5 . Then $M:=\operatorname{Bs} \Lambda$ is a union of $16 \mathbb{P}^{3}$ meeting in $\left\langle z_{1}, z_{2}, z_{3}\right\rangle$. Let $\Pi_{i}=\left\langle z_{1}, z_{2}, z_{3}, p_{i}\right\rangle$, then $\Pi_{i} \cap \Pi_{j}=\left\langle z_{1}, z_{2}, z_{3}\right\rangle$ and $\Pi_{i} \subset M$. By construction we have $\mathcal{L}_{7,3 \mid \Pi_{i}}^{H} \subset \mathcal{L}_{3,3}\left(2^{4}\right)$ on the other hand specialization can only increase dimension of linear systems, therefore

$$
\operatorname{dim}\left(\mathcal{L}_{7,3}^{H}\right)_{\mid \Pi_{i}} \geq \operatorname{dim} \mathcal{L}_{7,3}\left(2^{14}\right)_{\mid \Pi_{i}}=4,
$$

where the last equality is proved in Pos2, subsection 5.4]. Let $D_{1}, D_{2} \in \mathcal{L}_{7,3}^{H}$ be two general elements. Then $\left(D_{1} \cdot D_{2}\right)_{\mid \Pi_{i}}$ contains a twisted normal curve passing through $\left\{z_{1}, z_{2}, z_{3}, p_{i}\right\}$. Set $Q_{1}, \ldots, Q_{4} \in \Lambda$ general elements. Then the 1-cycle $Q_{1} \cap \ldots \cap Q_{4} \cap D_{1} \cap D_{2}$ contains rational curves intersecting in $\left\{z_{1}, z_{2}, z_{3}\right\}$ and it has positive genus. This, by Remark 11, shows that $g_{7}>0$. 
We collected all needed result to prove Theorem 2 .

Proof of Theorem 2, By [Me1, Theorem 4.3, Proposition 2.4] and [AC, Theorem $3.2]$ we may assume $d \leq n$ and $n \geq 4$. If $d=2$ and $\operatorname{dim} \mathcal{L}_{n, 2}\left(2^{h}\right)=n$ then the map associated to $\mathcal{L}_{n, d}\left(2^{h}\right)$ is always of fiber type.

If $d=3, n \equiv 0(3)$, and $n \geq 6$ then Theorem 7 forces $h=k(n)-1$. Then, by Equation (7) and Lemma 42, the sectional genus of $\mathcal{L}_{n, 3}\left(2^{h}\right)$ is positive. If $d=3$, $n \geq 7$ and $n \equiv 1(3)$ we conclude as before via Equation (7) and Lemma 43 that the sectional genus is positive. It is easy and well known that $\mathcal{L}_{4,3}\left(2^{6}\right)$ induces a fiber type map that contracts the rational normal curves through the 6 points. This analysis proves the theorem for $d \leq 3$.

Assume that $n \geq d \geq 4$. By Theorem [7 $h=k(n, d)-1$. If $n=d=4$, then $h=13$. There is a pencil of quadrics in $\mathbb{P}^{4}$ through 13 general points, so $\mathcal{L}_{4,4}\left(2^{13}\right)$ admits a linear subsystem of reducible divisors with base locus in codimension 2 , hence the associated map cannot be birational. Suppose then $(n, d) \neq(4,4)$. By Lemma 41] $\mathcal{L}_{0}(n, d)$ is a specialization of $\mathcal{L}_{n, d}\left(2^{h}\right)$ and it has positive sectional genus. This shows that $\mathcal{L}_{n, d}\left(2^{h}\right)$ does not define a Cremona modification.

\section{REFERENCES}

[AH] J. Alexander, A. Hirschowitz, The blown-up Horace method: application to fourth-order interpolation, Invent. Math. 107 (1992), no. 3, 585-602.

[AC] E. Arbarello, M. Cornalba, Footnotes to a paper of Beniamino Segre, Math. Ann. 256 (1981), no. 3, 341-362.

[AGMO] E. Angelini, F. Galuppi, M. Mella, G. Ottaviani, On the number of Waring decompositions for a generic polynomial vector, to appear on Journal of Pure and Applied Algebra, arXiv:1601.01869

[AHJKS] A. Anandkumar, D. Hsu, M. Janzamin, S. Kakade, When are overcomplete topic models identifiable? Uniqueness of tensor Tucker decompositions with structured sparsity, J. Mach. Learn. Res. 16 (2015), 2643-2694.

[BO] C. Brambilla, G. Ottaviani, On the Alexander-Hirschowitz Theorem, J. Pure Appl. Algebra, 212 (2008), 1229-1251.

[Br] J. Bronowski, The sum of powers as canonical expression, Mathematical Proceedings of the Cambridge Philosophical Society, 29 (1933), 69-82.

[COV1] L. Chiantini, G. Ottaviani, N. Vannieuwenhoven, An algorithm for generic and lowrank specific identifiability of complex tensors, SIAM J. Matrix Anal. Appl. 35 (2014), no. $4,1265-1287$.

[COV2] On generic identifiability of symmetric tensors of subgeneric rank, to appear in Trans. Amer. Math. Soc. arXiv:1504.00547

[Ch] W. Chow, On the genus of curves of an algebraic system, Trans. Amer. Math. Soc. 65, (1949). 137-140.

[Cil] C. Ciliberto, Geometric aspects of polynomial interpolation in more variables and of Waring's problem, Proceedings of the European Congress of Mathematics, Barcelona, July 10-14, 2000, Birkhauser, Progress in Math., 201, vol. 1, 289-316.

[CM] C. Ciliberto, R. Miranda, Degenerations of planar linear systems, Jurnal für die reine und angewandte Math., 501 (1998), 191-220.

[HOOS] J. Hauenstein, L. Oeding, G. Ottaviani, A. Sommese, Homotopy techniques for tensor decomposition and perfect identifiability, to appear in Journal für die reine und angewandte Mathematik, arXiv:1501.00090

[Hi] D. Hilbert, Letter adressée à M. Hermite, Gesam. Abh. vol II, 148-153.

[Lan] J. M. Landsberg, Tensors: geometry and applications. Graduate Studies in Mathematics, 128, American Mathematical Society, Providence, RI (2012). xx+439 pp.

[MM] A. Massarenti, M. Mella, Birational aspects of the geometry of varieties of sums of powers, Adv. Math. 243 (2013), 187-202.

[Me1] M. Mella, Singularities of linear systems and the Waring problem, Trans. Amer. Math. Soc. 358 (2006), no. 12, 5523-5538.

[Me2] Base loci of linear systems and the Waring problem, Proc. Amer. Math. Soc. 137 (2009), no. 1, 91-98.

[Ne] M. Nesci, Collisions of fat points, PhD thesis Università Roma III (2009). 
[Pa] F. Palatini, Sulla rappresentazione delle forme ternarie mediante la somma di potenze di forme lineari, Rom. Acc. L. Rend. 12 (1903), 378-384.

[Pos1] E. Postinghel, Degenerations and applications: polynomial interpolation and secant degree, $\mathrm{PhD}$ thesis Università di Tor Vergata Roma (2010).

[Pos2] _ A new proof of the Alexander-Hirshowitz interpolation theorem, Ann. Mat. Pura Appl. Vol. 191 (2012), 77-94.

[Ri] H.W. Richmond, On canonical forms, Quart. J. Pure Appl. Math. 33 (1904), 967-984.

[Sy] J.J. Sylvester, Collected works, Cambridge University Press (1904).

Dipartimento di Matematica e Informatica Università di Ferrara, Via Machiavelli 35, 44100 FERrARA ITALiA

E-mail address: glpfnc@unife.it, mll@unife.it 